\title{
Ethnicité et santé mentale : Conceptualisation, définition et opérationnalisation de l'ethnicité dans le contexte canadien
}

DE Clarke, Ph.D. (1-4); A Colantonio, Ph.D. (3-6); AE Rhodes. Ph.D. (2,3,7-9); M Escobar, Ph.D. (3)

\section{Résumé}

La présente étude fournit un compte rendu critique des études canadiennes sur l'ethnicité et la santé mentale pour ce qui est de la définition, de la conceptualisation et de l'opérationnalisation de l'ethnicité. Elle constitue un article fondateur sur les problèmes de méthodologie liés à ces facteurs et leurs répercussions, dans le but de guider la recherche future et de rendre possible la comparabilité des résultats entre les différentes études. Les bases de données Sociological Abstracts, PsycINFO, MEDLINE et CINAHL ont servi à répertorier les articles canadiens pertinents publiés entre janvier 1980 et décembre 2004. Ce compte rendu met en évidence un certain nombre de questions importantes que les futurs chercheurs devront prendre en considération, telles que la nécessité : 1) d'expliquer clairement pourquoi l'ethnicité est importante dans le cadre de leur recherche; 2) de fournir une définition claire de l'ethnicité, qui influe sur sa conceptualisation et son opérationnalisation; 3) de présenter une conceptualisation de l'ethnicité fondée sur la théorie, qui doit être reliée à la question sur laquelle porte la recherche et 4) de donner des justifications claires pour ce qui est des décisions prises au sujet de la source de données utilisée, de l'opérationnalisation de l'ethnicité et des catégories ethniques incluses dans leurs études.

Mots clés : ethnicité, origine ethnique, culture, race, santé mentale

\section{Introduction}

La population immigrante du Canada est originaire de partout dans le monde, mais compte un nombre de plus en plus grand d'immigrants provenant de l'Afrique, de l'Asie, des Caraïbes et du Moyen-Orient (soit des minorités visibles) ${ }^{1}$. Sa population autochtone culturellement diversifiée ajoute au mélange ethnique ${ }^{2}$. Le Canada s'enorgueillit d'être une société multiculturelle et reconnaît le droit de toute personne de s'identifier à ses antécédents culturels tout en suivant le mode de vie canadien $^{3-6}$. C'est ce qui a été défendu dans la politique législative de 1971 sur le multiculturalisme, qui mettait l'accent sur un traitement équitable pour tous, indépendamment de la race, de la couleur de la peau ou de l'ethnicité, en particulier pour ce qui est des possibilités d'accès à l'éducation et des possibilités du marché de l'emploi ${ }^{3-6}$. Des inégalités institutionnelles entre les groupes ethniques, y compris l'accès à l'éducation et au marché de l'emploi ainsi qu'aux soins de santé et de santé mentale, demeurent flagrantes ${ }^{7,8}$, ce qui peut avoir une incidence sur la santé mentale de la population ${ }^{2,9}$. Par exemple, les Autochtones continuent d'avoir une santé mentale moins bonne lorsqu'on la compare à celle de la population générale, et, au même titre que les minorités visibles, ils rencontrent constamment des difficultés en ce qui concerne l'accès à des soins de santé mentale adaptés à leur réalité culturelle?. Une meilleure compréhension de la relation entre l'ethnicité et la santé mentale est impérieuse et hautement pertinente pour les décideurs et les praticiens de la santé mentale dans le contexte canadien.

La documentation canadienne sur l'ethnicité et la santé mentale est plutôt limitée, et ce, malgré sa pertinence. Une grande partie des études existantes dans ce domaine ont été réalisées aux États-Unis (É.-U.) ${ }^{10-15}$ et au Royaume-Uni (R.-U.) ${ }^{16-19}$, et leur applicabilité au Canada est incertaine. Les conséquences sur le plan politique, social et économique associées à l'ethnicité ou à l'identité ethnique sont susceptibles de différer d'un pays à l'autre. Les différences dans la composition ethnique des pays, l'histoire de leurs politiques en matière d'immigration et leurs histoires de racisme ou d'esclavage ainsi que les catégorisations raciales ou ethniques font obstacle aux comparaisons entre les pays. L'histoire de l'esclavage et de la ségrégation a une très grande signification pour les groupes ethnoraciaux aux États-Unis ${ }^{2}$. En outre, les États-Unis sont vus comme une

\section{Coordonnées des auteurs}

1 Département de la santé mentale, Johns Hopkins Bloomberg School of Public Health, Baltimore, Maryland

2 Département de psychiatrie, Université de Toronto, Toronto (Ontario)

3 Département des études supérieures en sciences de la santé publique, Université de Toronto

4 Toronto Rehabilitation Institute, Toronto (Ontario)

5 Département de la science occupationnelle et de l'ergothérapie, Université de Toronto

6 Département des sciences de la réadaptation, Université de Toronto

7 Suicide Studies Unit, St. Michael's Hospital, Toronto (Ontario)

8 Inner City Health Research Units, St. Michael's Hospital

9 Institute for Clinical Evaluative Sciences, Sunnybrook \& Women's College Hospital, Toronto (Ontario)

Correspondance : Diana E Clarke, Department of Mental Health, Johns Hopkins Bloomberg School of Public Health, Room 802 , 624 N Broadway, Baltimore,

MD 21205, Tél. : 410-955-0416, Courriel : dclarke@jhsph.edu 
société où les immigrants sont assimilés par le phénomène du creuset, c'est-à-dire que l'on s'attend à ce que les immigrants s'adaptent au mode de vie à l'américaine plutôt qu'ils conservent leur culture ${ }^{3}$. Le Canada n'a pas eu la même histoire de ségrégation raciale et est considéré comme une mosaïque dans laquelle on encourage les immigrants à la fois à conserver leur milieu culturel unique et à faire partie de la société canadienne ${ }^{3,6,20}$. En outre, les Latino-Américains forment une plus grande partie de la population immigrante des États-Unis que du Canada ${ }^{2,3}$.

La date de l'arrivée au pays et la composition ethnique de la population immigrante ne sont également pas les mêmes au Royaume-Uni et au Canada. Avant 1962, les citoyens des pays qui étaient autrefois des colonies, comme la Jamaïque et l'Inde (des pays qui sont restés dans le Commonwealth), avaient reçu un libre accès au Royaume-Uni et étaient recrutés activement comme immigrants dans ce pays ${ }^{21}$. Pendant ce temps, la population immigrante du Canada provenait principalement de l'Europe, du Royaume-Uni et des ÉtatsUnis $^{22,23}$. Après 1971, au moment où le Royaume-Uni a modifié sa loi sur l'immigration, la majorité de sa population immigrante provenait de l'Europe et de l'Afrique du Sud ${ }^{21}$, tandis que la majorité des immigrants au Canada provenait de l'Asie, de l'Afrique, des Caraïbes, de l'Amérique latine et du Moyen-Orient ${ }^{23-26}$. Ces faits indiquent que les résultats fondés sur les données du Royaume-Uni ou des ÉtatsUnis pourraient ne pas être applicables au Canada.

En ce qui concerne l'opérationnalisation de l'ethnicité, il existe des variations dans la documentation sur l'ethnicité et la santé mentale. L'absence d'une définition claire de l'ethnicité et le débat continuel concernant la façon dont la variable devrait être conceptualisée pourraient expliquer ces variations ${ }^{27,28}$. D'autres facteurs comme la source de données utilisée, la faisabilité, le moment de la collecte des données, la région étudiée et la taille de l'échantillon ont aussi une incidence sur l'opération- nalisation de la variable. Actuellement, il n'existe aucun article canadien fondateur portant sur les problèmes de méthodologie liés à la définition, à la conceptualisation et à l'opérationnalisation de l'ethnicité et sur leurs répercussions, dont le but est de guider la recherche future et de rendre possible la comparabilité entre les études. Le présent article fournit un compte rendu critique des études canadiennes empiriques sur l'ethnicité et la santé mentale et met l'accent sur ces aspects. Un aperçu général de la définition et de la conceptualisation de l'ethnicité est fourni en premier lieu, afin de servir de guide pour le processus d'examen. L'accent a été mis sur les études quantitatives présentant des données sur les populations composées de plus d'un groupe ethnique.

\section{Méthodes}

Les bases de données bibliographiques Sociological Abstracts, PsycINFO, MEDLINE et CINAHL ont servi à répertorier les études empiriques originales pertinentes et de nature quantitative aux fins de ce compte rendu, grâce à l'application des termes de recherche des groupes 1 et 2, qui sont présentés dans la figure 1. Les études étaient retenues si : 1) elles traitaient d'ethnicité et de santé mentale; 2) elles renfermaient des données sur les Canadiens; 3 ) elles portaient sur plus d'un groupe ethnique; 4) elles avaient été publiées entre janvier 1980 et décembre 2004 et 5) elles étaient de nature quantitative.

Des rapports de synthèse et des chapitres généraux sur les théories et la définition de l'ethnicité non spécifiques au Canada ont servi à fournir une vue d'ensemble de la définition et de la conceptualisation de l'ethnicité. Ils ont également servi de guide à la critique des études sur l'ethnicité et la santé mentale répertoriées et examinées dans le présent document. Des articles sur l'ethnicité et les politiques d'immigration spécifiques au Canada ont été utilisés pour établir un contexte historique afin de faciliter la compréhension dela composition ethnique de la population canadienne au fil du temps et de ses incidences sur les études examinées.

\section{Resultats}

Après que tous les doubles ont été retirés, 49 études empiriques quantitatives portant sur deux groupes ethniques ou plus ont été retenues et incluses dans le présent compte rendu (figure 1), elles sont présentés dans l'annexe 1 . Vingt-sept études ${ }^{2,5,29-53}$ étaient fondées sur des enquêtes sur la population ou sur des collectivités et comportaient une analyse des données secondaires, 12 étaient des études de moindre envergure se servant d'échantillons non cliniques ${ }^{54-65}$ et 10 étaient des études de moindre envergure fondées sur des échantillons cliniques ou spécialisés ${ }^{66-75}$. L’année et le lieu de la publication, la taille de l'échantillon, les groupes ethniques inclus et une critique de la définition de l'ethnicité sont présentés dans l'annexe 1.

\section{Analyse}

\section{Définition}

L'absence de consensus sur la définition de l'ethnicitée,76-80 ressort des 49 études canadiennes sur l'ethnicité et la santé mentale ci-incluses ${ }^{2,5,29-75}$, parmi lesquelles aucune ne contient de définition explicite de cette variable. Il existe deux conceptions majeures quant à la façon dont l'ethnicité émerge : la conception primordialiste et la conception constructiviste 20,76,81 $^{\text {. Les }}$ primordialistes traditionnels considèrent l'ethnicité comme « une condition assignée, attribuée à la naissance, qui est plus ou moins fixe et permanente » [TRAducTION] $]^{20,76,81-85}$. Par conséquent, l'identité d'un individu est définie, entre autres, par les conditions biologiques, culturelles, politiques et économiques du groupe dans lequel il est né, que ce groupe en domine d'autres ou qu'il soit dominé par eux ${ }^{20,78,81,84}$. La conception primordialiste plus modérée met l'accent sur le fondement social et non biologique de l'ethnicité, reconnaissant 


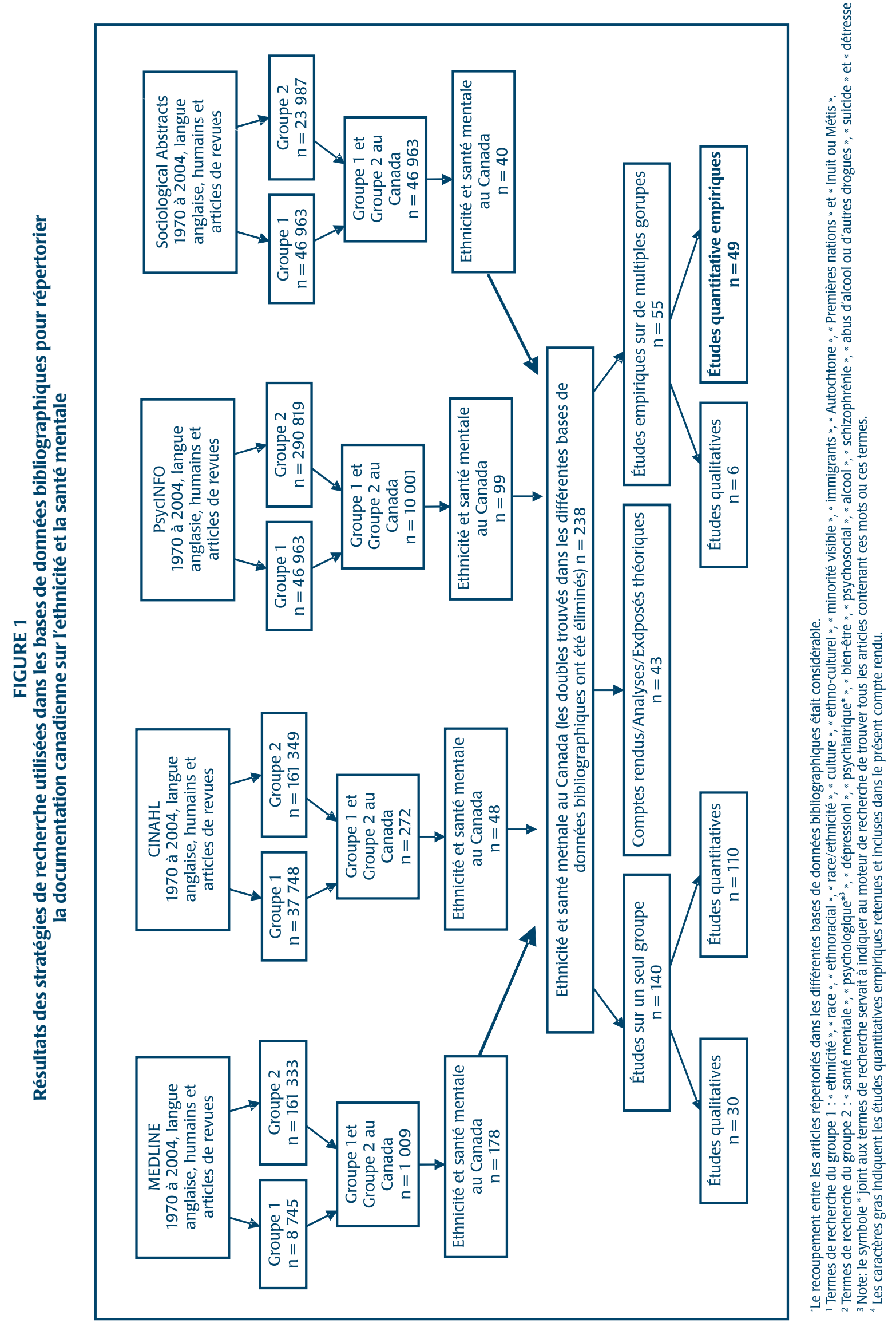


que l'identité ethnique peut également être construite socialement et fondée sur " les circonstances particulières ", étant ainsi "situationnelle, et non biologique " et " flexible, et non fixe " [Traduction $]^{81}$. Les constructivistes considèrent l'ethnicité comme " une construction sociale dont les principaux déterminants sont les facteurs écologiques et sociaux » [TRADUCTION] ${ }^{12,24-26}$. Selon Kaufman ${ }^{85}$, la distinction entre les deux conceptions est artificielle, et la définition de l'ethnicité devrait comprendre une synthèse des deux. Par conséquent, le thème sous-jacent à ce concept de l'ethnicité est le partage d'une culture commune, qui peut être fondée sur une combinaison de facteurs tels que la langue, la religion, l'identité nationale, les coutumes et la position sociale ou politique au sein du système social d'un pays ${ }^{81}$.

\section{Conceptualisation}

Il existe deux principaux points de vue quant à la façon dont l'ethnicité est conceptualisée : 1) l'identité ethnique, qui fait référence à l'auto-identification à un ou à des groupes culturels particuliers ${ }^{20,76,80,81}$ ou 2) l'origine ethnique, qui fait référence à la classification en fonction des groupes culturels ou ethniques auxquels les ancêtres de la personne appartiennent ${ }^{20}$. Les traits culturels spécifiques comme la langue, le nom de famille ou la région de naissance ont été utilisés à titre de variables de substitution pour l'origine ethnique dans certaines études.

D'un point de vue canadien, la politique du pays sur le multiculturalisme, qui a guidé l'élaboration des politiques gouvernementales depuis 1971 et qui défend la possibilité pour les individus de conserver leur milieu ethno-culturel ${ }^{8}$, prescrit que l'ethnicité soit constituée à la fois d'un « particularisme ethnoculturel » et d'une adhésion aux « valeurs » canadiennes ${ }^{76}$. Ce précepte se reflète dans la conceptualisation de l'ethnicité dans les enquêtes à l'échelle nationale, qui force les participants à s'identifier par leur milieu ancestral, peu importe depuis combien de temps ils sont au Canada. En outre, cette politique est responsable de la désignation de toutes les personnes qui ne sont pas des Autochtones et qui sont originaire de l'Afrique, de l'Asie, des Caraïbes, de l'Amérique latine et du Moyen-Orient, « qui ne sont ni caucasiens, ni blancs » [Traduction], en tant que minorités visibles ${ }^{1,3,6}$.

\section{Conceptualisation fondée sur l'identité ethnique}

L'identité ethnique peut être déterminée de façon interne ou externe ${ }^{20,78}$. L'identité ethnique interne désigne l'auto-identification d'un individu à un ou à des groupes ethniques particuliers ${ }^{78,86,87}$. Cette conceptualisation reflète le niveau d'adhésion et d'identification d'un individu aux valeurs, aux coutumes et aux idéologies d'une culture particulière $^{87}$, qui peut être dicté de l'intérieur ou de l'extérieur ${ }^{78,86-89}$. Les individus peuvent choisir de s'identifier à un ou à des groupes ethniques particuliers parce qu'ils croient à leurs coutumes, à leurs normes et à leurs idéologies et à cause du sens que cette identification donne à leur vie (c.-à-d. dicté de l'intérieur). Cependant, certaines circonstances dans l'environnement externe de l'individu, comme la discrimination fondée sur l'ethnicité et le sentiment d'être différent de la majorité ethnique, peut faire en sorte que certains individus s'identifient à des groupes ethniques particuliers en raison du sentiment de solidaritéet d'appartenance qu'ils peuvent éprouver dans ces groupes (c.-à-d. dicté de l'extérieur) ${ }^{78,80,87}$.

Étant donné que l'identité ethnique déterminée de façon externe se rapporte à la catégorisation par les autres en fonction de la nationalité ou de l'origine ethnique de la personne et ne reflète pas l'adhésion ou l'identification de cette personne à une ou à des cultures spécifiques ${ }^{15}$, elle fait l'objet d'une discussion dans le contexte de l'origine ethnique dans la section suivante. Ce genre d'attribution externe de l'ethnicité aux individus par la société dominante peut entrer en conflit avec leur adhésion à des groupes ethniques particuliers et peut être éprouvante. Mahtani ${ }^{6}$ l'illustre bien dans son entrevue avec des femmes d'origine raciale mixte faisant partie des minorités visibles nées au Canada, qui se sentent souvent forcées par la majorité ethnique de s'identifier à leur milieu ancestral lorsqu'on leur demande quelle est leur identité ethnique (c.-à-d. en leur posant la question : "mais d'où venezvous? »).

L'une des principales faiblesses de l'autoidentification de l'ethnicité est qu'elle est fondée sur la perception de la personne, qui est susceptible de changer selon la situation et au fil du temps et de produire des résultats différents selon le moment où se déroule l'enquête et selon la question de recherche sur laquelle porte l'enquête ${ }^{27,28}$. Cela justifie probablement l'absence d'une conceptualisation de l'ethnicité en fonction de l'identité ethnique dans les études, sauf pour ce qui est des études respectives de De Wit et coll. ${ }^{40}$, de De Wit et Beneteau ${ }^{41,42}$ et de Feldman et coll. ${ }^{43}$. De Wit et coll..$^{40-42}$ ont fait une analyse des données secondaires issues de l'Enquête sur la santé en Ontario de 1990, dans laquelle on a demandé aux répondants de choisir jusqu'à quatre catégories ethniques concordant avec leur "identité ethnique ou culturelle » [Traduction]. De Wit et Beneteau ${ }^{41,42}$ ont utilisé l'information sur la langue principale dont les répondants se servent à la maison afin d'aider à « mieux identifier les groupes ethniques » [TRADucTION]. Si les répondants indiquaient le français ou le français plus la langue d'autres groupes ethniques, mais qu'ils ne choisissaient pas le français comme langue maternelle, ils étaient alors reclassés comme anglophones ${ }^{41,42}$, ce qui indique une classification de l'identité ethnique dictée de l'extérieur. Cette classification de l'identité ethnique dictée de l'extérieur peut avoir divergé de la véritable identité ethnique de la personne. Si le niveau d'adhésion à des valeurs, à des coutumes et à des idéologies culturelles particulières est important en ce qui concerne la perception d'un trouble de santé mentale par la personne et les chances, par la suite, pour qu'elle le reconnaisse, en discute ou le signale, et il s'agit là d'une question fondamentale à laquelle on s'intéresse, alors l'utilisation de l'identité ethnique fournirait des renseignements plus appropriés. Feldman et coll. ${ }^{43}$ se sont servis de la question : "Veuillez inscrire le terme qui décrit le mieux le caractère ethnique de votre milieu de vie quotidien » [TRADUction], qui suppose une conceptualisation de la variable selon l'identité ethnique. 


\section{Conceptualisation fondée sur l'origine ethnique}

L'origine ethnique désigne la catégorisation des individus dans un ou des groupes ethniques d'après leurs origines ancestrales $^{78}$. La question « À quel(s) groupe(s) ethnique(s) ou culturel(s) vos ancêtres appartiennent-ils? » [TRADUction] a été utilisée pour déterminer l'origine ethnique $e^{90,91,84,95}$. Apparemment, cette méthode de conceptualisation serait plus objective et produirait des groupes ethniques bien définis et représentatifs de la population, mais elle ne prend pas en compte le niveau d'adhésion aux idéologies ou aux coutumes culturelles ${ }^{27,92,93}$.

La conceptualisation de l'ethnicité en fonction de l'origine ethnique a été observée dans certaines études canadiennes sur l'ethnicité et la santé mentale, notamment dans celles pour lesquelles les auteurs se sont servis de données issues d'enquêtes nationales ou régionales ${ }^{2,5,31,32,35,46-48,52}$, dont l'Enquête nationale sur la santé de la population (ENSP) ${ }^{90}$, l'Enquête sur la santé dans les collectivités canadiennes (ESCC) ${ }^{91}$, l'Enquête longitudinale nationale sur les enfants et les jeunes (ELNEJ) ${ }^{94}$, l'Enquête nationale sur le don, le bénévolat et la participation (ENDBP) ${ }^{95}$ et l'Étude longitudinale sur le vieillissement au Manitoba de $1971^{31}$. Dans le cadre de chaque enquête, on cherchait à obtenir des renseignements sur le ou les groupes ethniques auxquels appartenaient les ancêtres de la personne, et une liste de catégories ethniques permettant des choix multiples était fournie ${ }^{90,91,94,95}$. De ce fait, l'existence de groupes multiethniques, qui sont typiques du Canada ${ }^{8}$, a été reconnue et la fiabilité des groupes définis a été améliorée ${ }^{27}$. Fait révélateur de l'accent mis sur la politique du pays en matière de multiculturalisme, les répondants aux enquêtes qui se sont identifiés comme "Canadiens " devaient indiquer leur origine ancestrale, et ce, même s'ils étaient nés au Canada.

\section{Traits culturels spécifiques comme variables de substitution pour l'origine ethnique}

On pourrait présumer que les études qui ont eu recours à des données issues d'enquêtes dans lesquelles l'ethnicité était fondée sur l'origine ethnique conceptualiseraient l'ethnicité en conséquence; or, ce n'est pas le cas. $\mathrm{Ali}^{5}$ et $\mathrm{Ma}^{47}$ se sont servis du pays de naissance en songeant à l'ethnicité, malgré l'utilisation respective des données de l'ESCC ${ }^{91}$ et de l'ELNEJ ${ }^{94}$, ces deux enquêtes renfermant des questions spécifiques sur l'ethnicité. La justification de l'emploi du pays de naissance plutôt que des réponses aux questions sur l'ethnicité n'a pas été fournie, mais ce choix est vraisemblablement attribuable à l'utilisation de données à grande diffusion. Les versions à grande diffusion des enquêtes nationales canadiennes sont facilement accessibles pour les chercheurs, mais elles sont dénuées de renseignements uniques sur l'identification et ne contiennent pas les renseignements détaillés sur l'ethnicité et la culture que l'on trouve dans les versions à diffusion restreinte.

Penning ${ }^{32}$ s'est servie des données de la Social Change in Canada Survey (SCCS), dans laquelle le pays de naissance a servi à déterminer l'ethnicité. La détermination de l'ethnicité en fonction du pays de naissance dans cette étude met en lumière la faiblesse inhérente au fait d'effectuer l'analyse des données secondaires lorsqu'on doit se contenter des variables disponibles, quelles qu'elles soient. En gardant cela en tête, il est tout de même important de mentionner que, en raison de l'industrialisation et de la migration mondiales de plus en plus grandes, il est devenu inapproprié de se servir du pays ou de la région de naissance comme seul indicateur de l'origine ethnique. Une personne peut être née dans un pays, de parents provenant d'un autre pays, passer seulement quelques années dans le pays de naissance, puis retourner avec ses parents dans le pays où se trouve leur milieu ancestral, choisissant ainsi de s'identifier à sa culture ancestrale. Par conséquent, le pays ou la région de naissance, également utilisés par $\mathrm{Ali}^{5}$, Barnes et coll. ${ }^{35}, \mathrm{Ma}^{47}$, Cohen et MacLean ${ }^{51}$, Rousseau et coll. ${ }^{53,61}$ et Aubert et coll. ${ }^{65}$, entraînerait une classification inexacte de cette personne. La plupart des pays renferment de nombreux groupes ethniques, et la plupart des groupes ethniques sont constitués de membres nés dans différents pays de naissance. Le pays de naissance utilisé en tant que variable de substitution pour l'origine ethnique ne met pas en évidence ce genre de complexités, occasionnant vraisemblablement une mauvaise classification de certaines personnes. Cette possibilité n'exclut pas l'utilisation du pays ou de la région de naissance comme variable de substitution pour l'ethnicité, mais elle souligne la nécessité qu'il y ait des discussions détaillées sur les limites inhérentes à son utilisation et les conséquences pour l'interprétation des résultats.

La langue et le nom de famille ont servi de variables de substitution pour l'origine ethnique dans certaines études ${ }^{37,54-59}$, mais sont susceptibles de conduire à une mauvaise classification de certains individus. En se servant des noms de famille pour déterminer les groupes ethniques dans leur enquête sur les différences ethniques par rapport à la dépression chez les étudiants universitaires à Toronto, Dion et Giordano ${ }^{57}$ n'ont pas réussi à classifier correctement les non-Blancs ou non-Caucasiens ayant un nom de famille non ethnique. Si les membres de certains groupes ethniques spécifiques avaient tendance à avoir un nom de famille non ethnique, comme les Noirs des Caraïbes et les Sud-Asiatiques d'origine chrétienne ${ }^{57}$, et qu'il était plus ou moins probable que ces individus soient en dépression, leur exclusion aurait introduit un biais dans les résultats. Bagley ${ }^{58}$ a aussi utilisé les noms de famille et la langue, mais en combinaison avec une question non spécifiée sur l' « ethnicité » servant à conceptualiser l'ethnicité. Les noms de famille ont été utilisés au départ pour identifier les personnes âgées chinoises habitant à Calgary, en Alberta, et les recruteurs de l'étude ont fait des appels téléphoniques de suivi. Par ces appels, ils ont identifié les personnes admissibles parlant le cantonais, à qui ils ont demandé des renseignements sur leur ethnicité. Cette combinaison de facteurs a permis de combler les lacunes de l'utilisation des noms de famille seulement.

Walter $^{37}$ et Dion ${ }^{59}$ ont utilisé la langue comme variable de substitution pour l'ethnicité, ce qui peut avoir permis de classifier correctement certains groupes ethniques, mais pas d'autres (p. ex., classer dans le groupe des Chinois les personnes qui ont déclaré parler un dialecte chinois). 
La population anglophone est ethniquement hétérogène, car l'anglais constitue la langue maternelle des habitants de nombreux pays; c'est pourquoi il est incorrect de traiter le groupe comme s'il était homogène $\mathrm{e}^{59}$. De plus, bien que le français et l'espagnol soient la langue maternelle des habitants de la France de l'Espagne respectivement, ils sont aussi la langue maternelle des habitants de certains pays des Caraïbes et de l'Afrique. Le fait de présumer que toutes les personnes ayant adopté ces langues sont d'origine européenne serait inexact ${ }^{59}$. Différents groupes ethniques peuvent parler la même langue ou les membres d'un même groupe ethnique peuvent parler différentes langues, ce qui ne serait pas pris en compte si l'on choisissait la langue comme seule variable de substitution pour l'origine ethnique.

\section{Ethnicité, race ou ethnicité et race}

En examinant les études de Weekes et coll. ${ }^{71}$ et de Cohen et McLean ${ }^{51}$ respectivement, il est devenu apparent que la catégorisation était fondée sur la race, malgré l'utilisation du terme " ethnicité ». De plus, les chercheurs ${ }^{51,71}$ laissent entendre que la race et la culture sont un seul et même concept, et ce, malgré l'existence de nombreux articles théoriques indiquant le contraire es,76-79,85-99. $^{2}$. À titre d'illustration, Cohen et McLean ${ }^{51}$ ont utilisé les données de l'Enquête sociale générale (ESG) de 1999 dans laquelle les répondants devaient répondre à des questions sur leurs « antécédents culturels ou

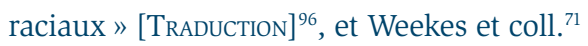
ont exprimé un intérêt à examiner à quel point leur échelle de résultats est " adaptée aux réalités culturelles » [TRAduction]. L'ethnicité désigne les caractéristiques culturelles communes telles que la religion, la langue, les coutumes et les origines ancestrales $^{20,76,78}$, tandis que la race désigne les caractéristiques physiques communes $^{28,65,78,86-101}$. Winker ${ }^{28}$, Williams ${ }^{77}$ et LaVeist $^{97}$ ont souligné l'importance d'établir une distinction entre la race et l'ethnicité ou la culture. La race est une mauvaise variable de substitution pour l'ethnicité, malgré le chevauchement de certaines caractéristiques ${ }^{28,97}$, et est discutable en tant que son unique indicateur dans les études respectives de Fry et Grover ${ }^{54}$ et de Devins et coll. ${ }^{74}$

$\mathrm{Wu}$ et coll. ${ }^{2}$ se sont servis de l'origine ethnique et du milieu racial pour créer des groupes ethnoraciaux. Cette façon de faire a permis l'identification de groupes de minorités visibles ${ }^{2}$ et a entraîné la reconnaissance de l'argument selon lequel des différences de pouvoir et de statut existent également entre les groupes raciaux ${ }^{77}$. Cette reconnaissance est importante, car " le statut dominant ou minoritaire du groupe traduit sa position à l'intérieur du système de stratification de la société » [TRADUCTION $]^{101}$, qui, à son tour, influe sur l'accès aux ressources sociales, politiques et économiques ${ }^{3,4,101}$. Les auteurs ${ }^{2}$ ont expliqué que leurs groupes ethnoraciaux correspondaient à la stratification sociale de la société canadienne (c.-à-d. la mosaïque verticale) $)^{2-4,7,8}$, ce qui leur a fourni un contexte qui a facilité l'interprétation des résultats obtenus. La mosaïque verticale renvoie à l'hypothèse selon laquelle les groupes ethniques sont intégrés de façon différentielle dans la société canadienne en fonction de l'histoire des politiques en matière d'immigration, qui, elle, est liée aux demandes changeantes dans le domaine des industries et de l'emploi au fil du temps ${ }^{2-4,7,8}$. Cette intégration différentielle dans la société canadienne a eu des incidences sur le statut socio-économique de ces groupes ${ }^{2-4}$, qui est significativement associé aux effets sur la santé mentale.

\section{Résumé}

Dans les études répertoriées, il semble que l'ethnicité ait été conceptualisée en fonction de l'origine ethnique par des questions sur l'ethnicité, par l'utilisation des traits culturels comme variables de substitution pour l'origine ethnique ou par la combinaison de l'origine ethnique et du milieu racial. Pour certaines de ces études, bien que le terme " ethnicité » ait été utilisé, la façon dont l'ethnicité a été conceptualisée et déterminée n'était pas claire ${ }^{30,33,34,54,55,70,75}$. Les lacunes intrinsèques découlant de l'utilisation de variables de substitution comme le pays de naissance, la langue ou le nom de famille mettent en évidence la nécessité d'établir l'ethnicité par des questions testées et validées de façon rigoureuse. Les questions visant à déterminer l'identité ethnique ou l'origine ethnique d'une personne ne permettent pas de savoir quel aspect de l'ethnicité est mis en avant par cette personne au cours d'une entrevue, et on ne sait pas non plus si la réponse peut être influencée par l'interviewer ou par la question qui est posée ${ }^{76}$. Cela fait ressortir la nécessité de présenter des justifications claires et fondées sur la théorie pour ce qui a trait à l'étude de l'ethnicité par rapport aux problèmes de santé mentale.

\section{Opérationnalisation}

L'opérationnalisation de l'ethnicité est importante pour l'interprétation des résultats de l'étude et pour permettre une comparaison entre différentes études. Cependant, cette opérationnalisation de l'ethnicité varie d'une étude à l'autre. Même les études ayant les mêmes sources de données et ayant conceptualisé l'ethnicité de manière semblable ont opérationnalisé la variable différemment. Parmi les facteurs ayant influencé ce phénomène se trouvent la diversité de la population étudiée, le moment et la région où la collecte de données a été effectuée, les restrictions quant à la taille de l'échantillon et le but de l'étude. Les effets de ces facteurs sont discutés dans les sections suivantes.

\section{Moment de la collecte de données et de l'étude}

Le moment où la collecte des données et où l'étude ont eu lieu a influencé l'opérationnalisation de l'ethnicité dans les études. Le moment où les données sont recueillies est lié aux modifications apportées à la politique en matière d'immigration au fil du temps, qui ont elles-mêmes été influencées par les changements économiques responsables des pénuries de maind'œuvre et du besoin en immigrants pour combler certaines possibilités d'emploi ${ }^{20,102}$. La politique canadienne précédente en matière d'immigration était fondée sur l'origine nationale et donnait la priorité 
aux immigrants de la Grande-Bretagne, de l'Europe et des États-Unis pour combler ces demandes en main-d'oeuvre ${ }^{22,25,103-105}$. Par conséquent, les immigrants de ces régions représentaient environ $95 \%$ de la population immigrante du Canada jusqu'en $1986^{22,25}$, et les études utilisant des données recueillies à cette époque ont une représentation inexistante ou quasi-inexistante des autres groupes ethniques $29,31,32,35,66,67$.

Le passage à un système de points d'appréciation universel en 1967 a été le moteur de l'accroissement de la diversité ethnique de la population canadienne, au sein de laquelle les immigrants européens constituaient $57 \%$ des immigrants en 1970, contre seulement $20 \%$ en $1996^{22,105}$. L'évolution de la composition ethnique de la population immigrante s'est produite graduellement : le petit nombre d'immigrants de l'Afrique, de l'Asie, des Caraïbes, de l'Amérique latine et du Moyen-Orient qui étaient présents au départ ont vu leurs proportions augmenter considérablement

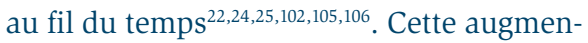
tation transparaît dans le fait que ces immigrants représentaient de 3 à $5 \%$ de la population canadienne jusqu'aux années 1980 comparativement à $73 \%$ entre 1996 et $2001^{107}$. Les variations dans les proportions de ces groupes d'immigrants les uns par rapport aux autres et par rapport aux immigrants en provenance de la GrandeBretagne, des États-Unis et de l'Europe ont été mises en évidence dans les études effectuées au moyen de données sur l'ethnicité recueillies au fil des ans. Les premières études ne contenaient aucun ou presque aucun groupe de minorités visibles $^{29,31,35,66,67}$, mais le nombre de ces groupes a augmenté dans les études suivantes $^{2,5,35,38,43,46-49,53,61-63}$. Ces groupes formaient des catégories distinctes ou non selon la variable étudiée et la nature de l'étude (c.-à-d. l'analyse de données secondaires au moyen de données à grande diffusion ne contenant aucun renseignement détaillé sur l'ethnicité). Dans des enquêtes sur des situations rares, comme certains problèmes de santé mentale, la petite taille des échantillons de groupes de minorités visibles particuliers a occasionné leur regroupement en une seule catégorie, ce qui n'a vraisemblablement pas permis de rendre compte de la perception des individus en ce qui a trait à leur identité ethnique, et ce qui a limité aussi l'interprétation des résultats de ce type d'études.

\section{Région de la collecte de données et de l'étude}

Les variations dans les modèles d'établissement des différents groupes ethniques au Canada ont donné lieu à des compositions ethniques différentes selon les régions géographiques. Ces compositions différentes ont eu une incidence sur l'opérationnalisation de l'ethnicité du point de vue géographique. Les modèles d'établissement des groupes d'immigrants ont toujours été liés aux endroits où se trouvent les possibilités d'emploi, elles-mêmes dictées par les changements dans l'économie au fil du temps ${ }^{78,102}$. Les premiers immigrants en provenance de la GrandeBretagne, de l'Europe et des États-Unis ${ }^{106}$ sont venus au Canada par suite de l'expansion rapide de l'Ouest canadien, rendue possible grâce à l'intégration de cette région aux marchés intérieurs et mondiaux et à la demande mondiale de blé $^{78}$. Par conséquent, bon nombre des premiers immigrants se sont établis dans les zones rurales de l'Ouest canadien pour combler la demande en ouvriers et en agriculteurs $^{66,102}$. Il y avait donc de fortes chances pour que les études menées dans l'Ouest canadien (p.ex., au Manitoba ${ }^{31,35,70}$, en Alberta ${ }^{66}$ et dans les Territoires du NordOuest ${ }^{67}$ ) contiennent une représentation significative des Britanniques, des Européens, des Nord-Américains et des Autochtones, mais presque aucune des groupes de minorités visibles.

Les immigrants plus récents en provenance de l'Asie, de l'Afrique, des Caraïbes, de l'Amérique latine et du Moyen-Orient étaient davantage susceptibles de s'établir à Montréal, à Toronto et à Vancouver ${ }^{102,108}$ parce qu'ils y voyaient de meilleures possibilités d'emploi correspondant à leurs compétences et à leur formation ${ }^{102}$. Des données à l'appui ont montré que seulement $58 \%$ des immigrants récents s'étaient établis dans ces régions en 1981, mais que le pourcentage a atteint $78 \%$ en $2001^{102}$. Des études récentes fondées sur des données concernant ces trois régions affichent des proportions variées d'ethnies faisant partie des minorités visibles $^{2,5,32,43,44,46-51,53-55,57-63,65,72,74,75}$. L'utilisation de données à grande diffusion a empêché l'examen de catégories ethniques très détaillés en raison de l'inaccessibilité de ce genre de renseignements.

\section{Le but de l'étude de recherche}

Tel que constaté dans les études respectives de Barnes et coll. ${ }^{35}$, de Lavallée et Bourgault ${ }^{45}$, de Seltzer et Langford ${ }^{67}$ et d'autres ${ }^{29,30,34,37,50,51,5}$ $8,61,64,66,69,70,73,75$, il y a une certaine souplesse par rapport à l'utilisation de catégories larges ou étroites au moment d'opérationnaliser l'ethnicité dans les études descriptives ou énumératives, en autant que ces catégories reflètent la composition ethnique de la population sur laquelle porte l'enquête ${ }^{20}$. Cependant, l'utilisation de catégories ethniques étroites fournit plus d'information ${ }^{20}$. En ce qui a trait aux études analytiques, l’opérationnalisation de l'ethnicité doit être fondée sur la théorie afin de fournir un cadre d'analyse et d'interprétation des résultats ${ }^{20}$. L'opérationnalisation de l'ethnicité dans les études analytiques examinées dans cet article $^{2,5,31,32,36-43,45-49,52,58,59,75}$ semble être fondée sur le système de stratification sociale au Canada, bien que ce ne soit pas toujours mentionné explicitement.

Les auteurs d'études intéressés à examiner l'état de santé mentale chez certains groupes ethniques avaient tendance à être clairs dans le choix et la définition de ces groupes, mais regroupaient souvent tous les autres groupes ethniques en un seul $^{30,31,33,34,36,38-42,44,45,51,54,61,67-71}$. Par exemple, Fry et Grover $^{54}$ souhaitaient examiner les problèmes de santé mentale des Asiatiques en comparaison avec ceux des Caucasiens et n'ont donc inclus que ces deux groupes dans leur étude. Pour leur part, Liban et Smart $^{30}$, Tonkin ${ }^{34}$, Beiser et coll. ${ }^{39}$, Borzecki et coll. ${ }^{68}$, Tcheng-Laroche et Prince ${ }^{33}$ ainsi que De Wit et Beneteau ${ }^{35,42}$ étaient principalement intéressés par la santé mentale des Indiens autochtones ${ }^{30,34,39,68}$ et des francophones $^{33,41,42}$ dans leurs études; ils ont donc choisi ces groupes de façon spécifique et ont réuni toutes les autres ethnies dans le groupe témoin.

Havens et Chappell, lorsqu'elles ont enquêté sur les effets de l'âge, du sexe et 
de l'ethnicité sur la santé mentale au Manitoba, se sont servies des groupes ethniques suivants : Nord-Américains, Britanniques, Français, Polonais/Russes/ Ukrainiens et " autres Européens $»^{31}$. Ces groupes étaient indicateurs du moment et de la région où l'étude a été menée. Les Nord-Américains, les Britanniques et les Français ont été parmi les premiers immigrants admis au Canada pour combler les demandes en main-d'œuvre d'après la politique d'immigration du pays à cette époque. Lorsque la demande en immigrants a augmentée2,106, on a accordé le droit d'entrée au Canada au groupe des " autres Européens ", qui comprenait les immigrants provenant de l'Allemagne, de la Norvège, du Danemark, de la Suède, de l'Islande, des Pays-Bas et de la Belgique ${ }^{31}$, au détriment des Polonais, des Russes et des Ukrainiens. Après la Seconde Guerre mondiale et avant la passage à la politique sur l'immigration consistant en un système de points d'appréciation universel, on a admis au Canada les immigrants en provenance de la Pologne, de la Russie, de l'Ukraine et d'autres pays de l'Europe de l'Est pour combler des possibilités d'emploi spécifiques et pour aider les survivants de $l^{\prime}$ Holocauste 22,106 . Les groupes ethniques inclus dans cette étude ${ }^{31}$ ont donc été intégrés à la hiérarchie sociale canadienne à différents niveaux et à différentes époques, mettant ainsi en évidence la ségrégation au sein de la société canadienne selon des critères ethniques ${ }^{22,104}$. Les Autochtones ont par ailleurs contribué à la diversité ethnique de la population manitobaine, en particulier dans les régions rurales $^{109}$, mais ils ont été exclus de l'étude afin de « réduire la partialité causée par les préjugés culturels »[Traduction $]^{31}$. Cet énoncé laisse entendre que les aspects culturels de l'ethnicité étaient importants pour ce qui est de la santé mentale et devaient être maintenus constants d'un groupe à l'autre. Il laisse aussi entendre que les catégories ethniques incluses dans cette étude étaient semblables entre elles sur le plan culturel, mais différentes de la catégorie des Autochtones ou que les groupes ethniques qui constituaient chacune des grandes catégories ethniques étaient semblables entre eux sur le plan culturel, mais que les groupes ethniques qui constituaient la grande catégorie des Autochtones étaient trop différents sur le plan culturel pour être combinés.

Penning ${ }^{32}$ a inclus des groupes ethniques semblables à ceux de Havens et Chappell ${ }^{31}$ dans son examen de la même hypothèse ayant comme objet le bien-être psychologique, et elle s'est servie d'un échantillon représentatif à l'échelle nationale de Canadiens âgés de 30 ans et plus. Les groupes reflétaient aussi la stratification sociale de la société canadienne. Havens et Chappell $^{31}$, contrairement à Penning ${ }^{32}$, ont constaté des différences ethniques en ce qui concerne la santé mentale, ainsi qu'un effet de " triple désavantage " relatif à l'âge, au sexe et à l'ethnie. Les problèmes méthodologiques liés aux différences dans la définition et la détermination des variables de santé mentale, à la conceptualisation (p. ex. l'utilisation du pays de naissance ${ }^{32}$ par opposition à une question spécifique au sujet de l'ethnie ${ }^{31}$ ) et à l'opérationnalisation de l'ethnicité, à la composition des populations étudiées, selon l'âge et de l'ethnie, et aux régions étudiées ont vraisemblablement joué un rôle dans les écarts observés.

Étant donné que Penning ${ }^{32}$ s'est servie du pays de naissance pour déterminer l'ethnicité et n'a mentionné l'exclusion d'aucun groupe ethnique, il est tenu pour acquis que les peuples autochtones et que toute personne faisant partie d'une minorité visible et étant née au Canada, quoiqu'en nombre minime, ont été inclus dans le groupe des Canadiens. Ce faisant, le groupe des Canadiens devient hétérogène et est différent des Canadiens inclus dans le groupe des Nord-Américains de l'étude de Havens et Chappell ${ }^{31}$. De plus, étant donné que Penning ${ }^{32}$ s'est servie de données provenant d'une enquête nationale, la catégorie ethnique « Autres » était probablement constituée des participants faisant partie de groupes de minorités visibles au Canada, tandis que ces groupes ont été exclus par Havens et Chappell ${ }^{31}$ en raison de la petite taille de l'échantillon. Penning ${ }^{32}$ a séparé les Canadiens et les Américains, mais elle a combiné les immigrants provenant de la France, de l'Allemagne, de la Norvège, du Danemark, de la Suède, de l'Islande, des Pays-Bas et de la Belgique dans le groupe des Européens du Nord. Ces facteurs ont nui à la capacité de comparer les résultats de ces deux études.

L'étude effectuée par $\mathrm{Ali}^{5}$ visait à comprendre la santé mentale de la population immigrante du Canada du point de vue de la dépression et de la dépendance à l'alcool. L'examen des différences ethniques au sein du groupe d'immigrants seulement ${ }^{5}$ n'était qu'un objectif secondaire. Les groupes ethniques examinés (c.-à-d.É.-U./Mexique, Amérique centrale et Amérique du Sud ainsi que Caraibes, Europe, Afrique et Asie) en fonction de la région de naissance reflétaient les différences dans le moment de l'entrée au Canada pour les divers groupes d'immigrants, ainsi que leur intégration différentielle au sein de la hiérarchie sociale du pays. Cela a fourni un cadre d'analyse et d'interprétation des résultats. La désagrégation du seul groupe des immigrants en fonction de l'ethnie porte à croire que l'enquête sur la variation de la santé mentale selon l'ethnie était importante uniquement pour les groupes d'immigrants et non pour les personnes nées au Canada ou que toutes les personnes nées au Canada ont vécu des expériences semblables qui peuvent avoir eu une incidence sur leur santé mentale. Étant donné que le groupe de personnes nées au Canada était probablement constitué de nombreux Canadiens de première génération ayant vraisemblablement vécu des expériences semblables à celles de leurs parents immigrants, il est bien possible que ces suppositions aient été inexactes. Compte tenu du rapport signalant une mauvaise santé mentale de la population autochtone au Canada ${ }^{8}$, il n'est peut-être pas approprié de combiner cette population au groupe des personnes nées au Canada. L'emploi de la région de naissance a de nombreux inconvénients et pourrait avoir créé un biais dans les résultats obtenus, mais il est probable que cette variable ait été utilisée en raison du manque d'informa- 
tions relatives à l'ethnicité dans l'ensemble de données de la version à grande diffusion du Cycle 1.1 de l'ESCC, que l'on présume être la source de données utilisée par $\mathrm{Ali}^{5}$.

Dans son examen des problèmes comportementaux et émotionnels des enfants immigrants par rapport à ceux des enfants non immigrants au Canada, $\mathrm{Ma}^{47}$ a considéré l'ethnicité comme une variable pertinente, mais seulement pour le groupe des immigrants. Les enfants immigrants ont été divisés en plusieurs groupes ethniques en fonction de leur région de naissance, y compris ceux qui proviennent des ÉtatsUnis, de l'Europe, de l'Asie et d'autres régions ${ }^{47}$, probablement en raison de l'utilisation de la version à grande diffusion de l'ELNEJ $^{94}$. Ces catégories ethniques sont différentes de celles qui ont été employées par $\mathrm{Ali}^{5}$, et ce, malgré l'utilisation de la même méthode de conceptualisation de l'ethnicité. Dans ces deux études, les hypothèses testées étaient différentes, et les populations et les variables étudiées étaient différentes, ce qui a nui à la capacité de comparer les résultats entre les études.

Beiser et coll. ${ }^{46}$ ont utilisé les données de l'ELNEJ $^{94}$ pour examiner la pauvreté familiale et les problèmes émotionnels et comportementaux chez les enfants immigrants par rapport aux enfants nés au Canada, et la variable de contrôle employée était l'ethnicité. Au contraire de ce qu'a fait $\mathrm{Ma}^{47}$, l'ethnicité a été examinée pour la totalité de l'échantillon ${ }^{46}$, ce qui a permis d'éliminer les ambiguïtés observées dans l'interprétation des résultats des études précédentes. Bien qu'une source de données fournissant des renseignements sur diverses catégories ethniques ait été utilisée dans le cadre de cette étude, l'ethnicité a été opérationnalisée à l'aide de quatre grandes catégories, soit les Blancs/Européens, les Asiatiques, les Noirs et les autres ${ }^{46}$. Cette façon de faire semble indiquer un intérêt pour un effet fondé sur le statut de minorité visible, ou une anticipation de cet effet. Les Autochtones vivant en dehors des réserves étaient aussi inclus dans l'ELNEJ ${ }^{94}$, et, puisqu'il n'y a aucune indication explicite de leur exclusion, on suppose qu'ils ont été combinés à la catégorie «Autres » ou qu'ils ont été inclus dans la catégorie "Blancs/Européens ». Le bien-fondé de l'inclusion des enfants autochtones dans l'une ou l'autre de ces catégories est discutable, étant donné les différences importantes sur le plan socioculturel.

Mata $^{48}$ a utilisé les données de l'ENDBP 95 pour examiner la satisfaction des groupes ethniques à l'égard de la vie au Canada et pour tester l'hypothèse selon laquelle toute variation peut être expliquée par les différences de statut socio-économique entre les ethnies. Dix-neuf catégories ethniques mutuellement exclusives ont été établies pour cette étude, et les limitations concernant la taille des échantillons ont mené au regroupement de différents groupes ethniques dans de plus grandes catégories (p. ex. les groupes ethniques des " Noirs ", des "Sud-Asiatiques ", des "Italiens » et des " Portugais » ont été regroupés dans les catégories des "Noirs/ Sud-Asiatiques » et «Italiens/Portugais ») ${ }^{48}$. La distinction entre certaines autres catégories ethniques n'était pas claire. Par exemple, les catégories des "Européens ", des «Italiens/Portugais », des "Allemands », des " Français », des "Ukrainiens », des "Polonais », etc., tous définitivement d'origine européenne, ont été utilisées sans qu'une justification claire ne soit fournie. La combinaison non justifiée de catégories ethniques larges et de catégories ethniques étroites a limité l'interprétation et la comparabilité des résultats. Il aurait été avantageux de se fonder sur un cadre théorique pour la division des catégories ethniques de façon à donner plus de signification aux résultats et à diminuer l'ambiguïté observée ${ }^{20}$.

Wu et coll. ${ }^{2}$ ont testé l'hypothèse selon laquelle les différences ethniques en ce qui concerne la dépression peuvent être expliquées par les variations relatives au statut socio-économique et à l'accès au soutien social (données issues de l'ENSP ${ }^{90}$ de 1996). Les groupes " ethnoraciaux » inclus dans cette étude étaient les suivants : " Asiatiques de l'Est/du Sud-Est », «Chinois », "Sud-Asiatiques », " Autochtones », « Noirs", " Arabes/Asiatiques de l'Ouest ", " LatinoAméricains », « Juifs », «Français », « Anglais », « autres Blancs » et " race mixte ». Ces groupes étaient « représentatifs de la stratification sociale de la société canadienne » [Traduction] et reflétaient les différences relatives au statut socio-économique et à l'accès au soutien social ${ }^{2}$. La désagrégation partielle du groupe des Asiatiques en " Asiatiques de l'Est/du Sud-Est » et " Chinois » est probablement attribuable à la présence écrasante des Chinois (environ 56 \% ) dans le groupe, qui aurait embrouillé l'interprétation de toute association avec la dépression. Une justification claire n'a pas été fournie pour cette désagrégation partielle; on a plutôt laissé le soin aux lecteurs de la présumer. La désagrégation d'autres groupes ethniques comme les Noirs et les Sud-Asiatiques n'a pas pu être effectuée en raison de la taille insuffisante de l'échantillon².

Wang et El-Guebaly ${ }^{52}$ ont opérationnalisé l'ethnicité au moyen de la dichotomie Blancs/non-Blancs, et ce, malgré leur utilisation des données de l'ENSP90 de 1996. Cette façon de procéder est probablement attribuable à l'utilisation des données de la version à grande diffusion de l'Enquête, quoique ce ne soit pas mentionné de manière explicite. Cette dichotomie, bien qu'elle soit pratique pour séparer les minorités visibles de la majorité ethnique, a été critiquée parce qu'elle ne donnerait pas une image claire des différences ethniques en matière de santé mentale $2,27,104$. Il est nécessaire d'améliorer cette dichotomie afin qu'elle rende compte des différences culturelles ou écologiques au sein de ces grands groupes ethniques ${ }^{104}$, lesquelles pourraient comprendre des préjugés culturels lors de l'étude des problèmes de santé mentale. Bien que les ethnies particulières formant le vaste groupe des " non-Blancs » aient une langue, une histoire, des coutumes et une mobilité sociale propres à chacune ${ }^{2}$, la discrimination en matière d'éducation et d'emploi fondée sur le statut de minorité visible $2,22,103,109$ justifie d'une certaine façon le fait de combiner ces groupes dans cette catégorie. L'emploi d'une catégorie ethnique aussi vaste est approprié si les chercheurs font valoir que c'est l'expérience commune d'être "non-Blanc » qui a une incidence sur la santé mentale, bien plus que l'effet des différences culturelles entre les perceptions et l'attitude des individus à l'égard 
des problèmes de santé mentale ${ }^{98}$, mais ceci n'était pas explicite.

Dion et Giordano ont inclus les « AngloCeltiques », les " Nord-Européens », les "Sud-Européens », les " Européens de l'Est», les " Asiatiques de l'Est » et les "SudAsiatiques » dans leur étude ${ }^{57}$. On dit que ces groupes ethniques correspondent à la composition ethnique de la population de l'Université de Toronto de $1988^{57}$ et reflètent les différences d'expérience en matière d'immigration, de condition économique et sociale à l'entrée et d'adaptation à la vie au Canada par rapport aux conflits entre les parents, ainsi que la probabilité de percevoir une discrimination économique ${ }^{57}$. D’après cette étude, les "Sud-Asiatiques » et les «Sud-Européens » sont plus susceptibles de faire une dépression que les "Anglo-Celtiques » et que les "NordEuropéens » en raison de la plus haute probabilité qu'il y ait des conflits entre les parents et qu'une discrimination économique soit perçue ${ }^{57}$. L'ethnicité a été déterminée en fonction du nom de famille ${ }^{57}$, ce qui a probablement entraîné une classification erronée des "Noirs » et des "SudAsiatiques » provenant d'un milieu chrétien. Comme les «Sud-Asiatiques » constituaient un groupe ethnique pertinent dans cette étude ${ }^{57}$, cette classification erronée a vraisemblablement eu une incidence sur les résultats obtenus parce qu'elle a atténué la mesure de l'effet et a miné l'applicabilité des résultats à l'ensemble des "SudAsiatiques » de Toronto. Dion et Giordano ${ }^{57}$ ont expliqué que, dans une étude antérieure portant sur 300 étudiants de cette même université, seuls $3 \%$ d'entre eux avaient indiqué être d'origine antillaise et que, par conséquent, une classification erronée possible selon le nom de famille ne créait que peu ou pas de biais dans leurs résultats. Cette explication suppose à tort que parmi les "Sud-Asiatiques », seuls ceux d'origine antillaise possèdent des noms de famille ne traduisant pas leur origine ethnique. La population chrétienne en Inde constitue environ $2,3 \%$ de la population totale du pays selon les recensements de 1991 et de $2001^{110}$.

\section{Résumé}

En résumé, l'opérationnalisation de l'ethnicité dans les études canadiennes existantes diffère selon la manière dont la variable est conceptualisée, les sources de données utilisées, le moment et la région où la collecte de données a été effectuée, le but de l'étude et le fait que l'étude comprenait ou non une analyse des données secondaires faite au moyen des versions à grande diffusion des enquêtes nationales. Dans certaines études, les auteurs ont tenté de désagréger les catégories ethniques, mais ont été confrontés à des tailles d'échantillons insuffisantes; il en a résulté que certains groupes ethniques distincts ont été réunis, tandis que d'autres groupes demeuraient désagrégés, et ce, sans justification claire. Des tailles d'échantillons insuffisantes ont été constatées dans les ensembles de données issus des enquêtes nationales, en particulier pour ce qui est des groupes ethniques formant des minorités visibles, mettant ainsi en évidence la nécessité qu’il y ait surreprésentation de ces groupes dans les enquêtes futures sur la population qui vise à fournir de l'information sur la santé ou la santé mentale de la population canadienne. Cette surreprésentation permettrait un examen des différences entre les groupes de minorités visibles pour ce qui a trait à la santé mentale, ce qui est important pour la planification de programmes en santé mentale afin de mieux servir la population multiethnique du Canada.

\section{Conclusions}

La présente critique de la définition, de la conceptualisation et de l'opérationnalisation de l'ethnicité dans les études canadiennes sur l'ethnicité et la santé mentale a soulevé un certain nombre de considérations essentielles, que nous formulons ci-dessous.

a) Le mot " ethnicité » est un terme complexe et abstrait pour lequel on n'a pas encore établi une définition unique et généralement acceptée. Cependant, le thème sous-jacent à ce concept de l'ethnicité est le partage d'une culture commune. Parmi les études, aucune n'a fourni de définition de l'ethni-cité a priori, probablement en raison de l'absence de consensus au sujet de cette définition. Par conséquent, tel que suggéré par les arbitres scientifiques des États-Unis ${ }^{28,77,79}$ et du Royaume-Uni ${ }^{27,92,93}$, il faudra expliquer clairement pourquoi l'ethnicité est importante dans le cadre d'une étude donnée.

b) L'examen de la relation entre l'ethnicité et la santé mentale doit être encouragé par une meilleure infrastructure, qui se traduit par un financement plus généreux, compte tenu de la pertinence du sujet dans le contexte canadien. L'analyse des données secondaires est très utile dans ce genre de travaux de recherche. Il est donc vital de faire en sorte que des données détaillées sur l'ethnicité soient plus facilement accessibles aux chercheurs tout en assurant le respect de la vie privée des participants aux enquêtes.

c) Certains chercheurs semblent avoir utilisé les données des versions à grande diffusion des enquêtes, ce qui a nui à leur capacité de désagréger les groupes ethniques en raison de l'absence de renseignements détaillés à ce sujet dans les fichiers. L'utili-sation des versions à grande diffusion doit être mentionnée explicitement, et ses limitations inhérentes doivent faire l'objet d'une discussion.

d) L'ethnicité peut être conceptualisée en fonction de l'origine ethnique, de l'identité ethnique ou d'une combinaison de l'origine ethnique et de la race. Chacune de ces méthodes a ses forces et ses faiblesses. La conceptualisation de l'ethnicité doit être fondée sur un cadre théorique, et elle doit avoir un rapport avec la question sur laquelle porte la recherche. Dans les études répertoriées, l'ethnicité était conceptualisée d'après l'origine ethnique, que ce soit par l'utilisation de questions spécifiques ou de variables de substitution comme la langue, le nom de famille ou le pays de naissance. Le pays de naissance, la langue et le nom 
de famille ont tendance à produire une classification erronée de certains groupes ethniques et des résultats biaisés lorsque le taux d'erreur diffère d'un groupe de résultats à l'autre. Cela n'exclut pas leur utilisation comme variables de substitution pour l'ethnicité; par contre, les chercheurs devront en indiquer les limites inhérentes et les conséquences en ce qui a trait aux résultats.

e) L'ethnicité fondée sur l'origine ethnique peut être plus stable si on fournit aux participants une liste de catégories ethniques et qu'ils peuvent choisir plus d'une catégorie $^{27,93}$. Cette liste doit être mise au point à partir d'une étude préliminaire sur le terrain, menée dans le but de déterminer les catégories ethniques courantes. Une option de réponse " autre » devrait être incluse de façon à ne pas limiter les choix des personnes. Cette liste doit être jointe en annexe aux résultats de la recherche ou doit être rendue disponible sur demande afin de faciliter la reprise de l'étude et de permettre aux lecteurs de déterminer la représentativité des catégories ethniques dans la population générale.

f) L'opérationnalisation de l'ethnicité variait entre des catégories ethniques très larges et d'autres très étroites, et ce, même si l'ethnicité était toujours conceptualisée de façon semblable ou que l'on utilisait toujours la même source de données. Ces variations étaient liées à l'absence d'une définition claire de l'ethnicité, à des différences entre le moment et la région où les études et les collectes de données ont eu lieu, au but des études et à l'utilisation de données à grande diffusion. Les chercheurs sont encouragés à fournir un résumé clair des décisions relatives à la source de données utilisée (en particulier pour ce qui est de l'analyse des données secondaires), à l'opérationnalisation de l'ethnicité et aux catégories incluses dans leur étude. Cela rendra plus faciles l'interprétation des résultats et les tentatives de reproduire les résultats de la recherche.

g) Les différences dans les variables étudiées et dans la façon dont ces variables sont mesurées ainsi que la variabilité des variables faisant l'objet d'un contrôle constituent des facteurs supplémentaires ayant eu une incidence sur la capacité de comparer les résultats entre les études. D’où la nécessité pour les chercheurs de définir clairement les variables qu'ils cherchent mesurer et de résumer les forces et les faiblesses des mesures utilisées pour calculer ces variables et les autres variables pertinentes.

\section{Remerciements}

Diana E. Clarke est associée de recherche du Département de la santé mentale de la Johns Hopkins Bloomberg School of Public Health et détentrice d'une bourse de recherche en études sur la santé de la population du Département de psychiatrie de l'Université de Toronto. Le financement des activités de $\mathrm{M}^{\mathrm{me}}$ Clarke provient d'une bourse de recherche postdoctorale des instituts de recherche en santé du Canada (subvention $n^{\circ}$ 200602MFE-159564-115967), de la Fondation du Toronto Rehabilitation Institute et en partie d'une bourse de recherche sur la santé de la population octroyée par le Département de psychiatrie de l'Université de Toronto. La présente étude a été financée en partie par le ministère de la Santé et des Soins de longue durée et par la Fondation ontarienne de la santé mentale.

\section{Références}

1. Statistique Canada. Guide de l'utilisateur du Recensement de 2001 : minorité visible et groupe de population. URL : http:// www12.statcan.ca/francais/census01/ Products/Reference/tech_rep/vismin.cfm.

2. Wu Z, Noh S, Kaspar V, Schimmele CM. Race, Ethnicity, and Depression in Canadian Society. J Health Social Behav. 2003;44:426-441.

3. Esses VM, Gardner RC. Multiculturalism in Canada: Context and Current Status. Canadian Journal of Behavioral Science. 1996;28:145-152.
4. Li PS. " Social Inclusion of Visible Minorities and Newcomers: The Articulation of "Race" and "Racial" Difference in Canadian Society ", préparé pour la Social Inclusion Conference de 2003: What Do We Know and Where Do We Go: Building a Social Inclusion Research Agenda, Ottawa, 27-28 mars 2003. URL : http:// www.ccsd.ca/events/inclusion/papers/ peter_li.pdf.

5. Ali J. La santé mentale des immigrants au Canada, supplément aux Rapports sur la santé, volume 13, 2002, p. 1-13.

6. Mahtani M. "Interrogating the hyphennation: Canadian multicultural policy and "mixed race" identities ", dans Hier SP and Bolaria BS (dir.), Identity and Belonging: Rethinking race and ethnicity in Canadian society, Toronto (Canada), Canadian Scholars' Press Inc, 2006, Chapitre 2, p. 31-42.

7. Porter J. The Vertical Mosaic: An Analysis of Social Class and Power in Canada, Toronto, University of Toronto Press, 1965, p. 60-103.

8. Lian JZ, Matthews DR. Does the Vertical Mosaic Still Exist? Ethnicity and Income in Canada, 1991. Can Rev Sociol Anthropol. 1998;35:461.

9. Kirmayer LJ, Minas $\mathrm{H}$. The future of cultural psychiatry: An international perspective. Can J Psychiatry. 2000;45:438-446.

10. Centers for Disease Control and Prevention. Suicide among Black youths - United States, 1980-1995. MMWR Morb Mortal Wkly Rep. 1998;47:106-193.

11. Shiang J. Does culture make a difference? Racial/ethnic patterns of completed suicide in San Francisco, CA 1987-1996 and clinical implications. Suicide Life Threat Behav. 1998,28:338-354.

12. Joe S, \& Kaplan MS. Suicide Among African American Men. Suicide Life Threat Behav. 2001;Vol.31:S1106-121. 
13. Oquendo MA, Ellis SP, Greenwald S, Malone KM, Weissman MM, Mann JJ. Ethnic and sex differences in suicide rates relative to major depression in the United States. Am J Psychiatry. 2001;158:1652-1658.

14. Price JH, Dake JA, Kuchareswki R. Assets as predictors of suicide attempts in African American inner-city youths. Am J Health Behav. 2001;25:367-375.

15. O’Donnell L, O’Donnell C, Merrit Wardlaw D, Stueve A. Risk and resiliency factors influencing suicidality among African American and Latino youth. Am J Community Psychol. 2004;33:37-49.

16. Neeleman J, Mak V, Wessely S. Suicide by age, ethnic group, coroners' verdicts and country of birth: A three year survey in Inner London. $\mathrm{Br} \mathrm{J}$ Psychiatry. 1997; 171:463-467.

17. Neeleman J, Wessely S. Ethnic minority suicide: A small area geographical study in South London. Psychol Med. 1999; 29:429-436.

18. Bhugra D, Desai M, Baldwin D. Attempted suicide in west London: Rates across ethnic communities. Psychol Med, 1999; 29:1125-1130.

19. McKenzie K, van Os J, Samele C, van Horn E, Tattan T, Murrau R. Suicide and attempted suicide among people of Caribbean origin with psychosis living in the UK. Br $\mathrm{J}$ Psychiatry. 2003;183:40-44.

20. Isajiw WW. Definitions of Ethnicity. Ethnicity. 1974;1:111-124.

21. Bell, BD. The Performance of Immigrants in the United Kingdom: Evidence from the GHS. The Economic Journal. 1997; 107:333-344.

22. Green AG, Green DA.. "The Economic Goals of Canada's Immigration Policy, Past and Present ", Department of Economics, University of British Columbia, 1996.

23. Li PS. "Race and ethnicity ", dans Li PS (dir.), Race and Ethnic Relations in Canada, Second Edition, Oxford University Press, Canada, 1999, p. 3-20.
24. Beaujot R. Immigration et données démographiques canadiennes : état de la recherche, Citoyenneté et Immigration Canada, 1998. URL : http://www.cic.gc.ca/ francais/ressources/recherche/index.asp.

25. Citoyenneté et immigration Canada. Statistiques sur la citoyenneté et l'immigration, 1966-1996, 2004. URL : http://epe.lac-bac. gc.ca/100/202/301/immigration_statisticsef/index.html.

26. Belanger A, Malenfant EC. Projections de la population des groupes de minorités visibles, Canada, provinces et régions, Ottawa, Statistique Canada, Division de la démographie, 2005, $\mathrm{n}^{\circ}$ 91-541-XIF au catalogue.

27. Aspinall PJ. Operationalising the collection of ethnicity data in studies of the sociology of health and illness. Sociol Health Illn. 2001;23:829-862.

28. Winker MA. Measuring Race and Ethnicity: Why and How? JAMA. 2004;292:1612-1614.

29. Murphy HBM. "European cultural offshoots in the New World: Differences in their mental hospitalization patterns. Part II: German, Dutch, and Scandinavian influences ", Archiv fur Psychiatrie und Nervenkrankheiten (Archives of Psychiatry and Neurological Sciences), vol. 228 (1980), p. $161-174$.

30. Liban CB, Smart RG. Drinking and drug use among Ontario Indian students. Drug Alcohol Depend. 1982;9:161-171.

31. Havens B, Chappell NL. Triple Jeopardy: Age, Sex, and Etnicity. Canadian Ethnic Studies. 1983;15:119-129.

32. Penning MJ. Multiple Jeopardy: Age, Sex, and Ethnic Variations. Canadian Ethnic Studies. 1983;15:81-105.

33. Tcheng-Laroche F, Prince R. Separated and divorced women compared with married controls: Selected life satisfaction, stress and health indices from a community survey. Soc Sci Med. 1983;17:95-105.
34. Tonkin RS. Suicide methods in British Columbian adolescents. J Adolesc Health Care. 1984;5:172-177.

35. Barnes GE, Currie RF, Segall A. Symptoms of Depression in a Canadian Urban Sample. Can J Psychiatry. 1988;33:386-392.

36. Sack WH, Beiser M, Phillips N, BakerBrown G. Co-morbid symptoms of depression and conduct disorder in First Nations children: Some findings from the Flower of Two Soils Project. Cult Med Psychiatry. 1993;16:471-486.

37. Walters, V. Stress, Anxiety and Depression: Women's Accounts of their Health Problems. Soc Sci Med. 1993;36:393-402.

38. Beiser M, Cargo M, Woodbury MA. A comparison of psychiatric disorder in different cultures: Depressive typologies in Southeast Asian refugees and resident Canadians. Int J Methods Psychiatr Res. 1994;4:157-172.

39. Beiser M, Sack W, Manson SM, Redshirt R, Dion R. Mental and the academic performance of First Nations and majorityculture children. Am J Orthopsychiatry. 1998;68:455-467.

40. De Wit ML, Embree BG, De Wit DJ. Determinants of the risk and timing of alcohol and illicit drug use onset among natives and non-natives: Similarities and differences in family attachment. Soc Biol. 1999;46:100-121.

41. DeWit DJ, Beneteau B. Predictors of the prevalence of alcohol use and related problems among Francophones and Anglophones in the province of Ontario, Canada. Journal of the Study of Alcoholism. 1998;59:78-88.

42. DeWit DJ, Beneteau B. Predictors of the prevalence of tobacco use among Francophones and Anglophones in the province of Ontario. Health Educ Res. 1999;14:209-223.

43. Feldman L, Harvey B, Holowaty P, Shortt L. Alcohol use beliefs and behaviors among high school students. J Adolesc Health. 1999;24:48-58. 
44. Beiser M, Dion R, Gotowiec A. The structure of attention-deficit and hyperactivity symptoms among Native and NonNative elementary school children. J Abnorm Child Psychol. 2000;28:425-437.

45. Lavallee C, Bourgault C. The health of Cree, Inuit and Southern Quebec Women: Similarities and Differences. Can J Public Health. 2000; 91:212-216.

46. Beiser M, Hou F, Hyman I, Tousignant M. Poverty, Family Process, and the Mental Health of Immigrant Children in Canada. Am J Public Health. 2002;92:220-227.

47. Ma X. The First Ten Years in Canada: a Multi-Level Assessment of Behavioral and Emotional Problems of Immigrant Children. Canadian Public Policy. 2002;28:395-418.

48. Mata F. A Look at Life Satisfaction and Ethnicity in Canada. Canadian Ethnic Studies. 2002;34: 51-63.

49. Wu Z, Hart R. Social and health factors associated with support among elderly immigrants in Canada. Research on Aging. 2002;24:391-412.

50. Blackstock C, Trocme N, Bennett M. Child maltreatment investigations among Aboriginal and Non-Aboriginal families in Canada. Violence Against Women. 2004; 10:901-906.

51. Cohen MM, MacLean H. Violence against Canadian Women. BMC Women's Health. 2004;4:S22.

52. Wang JW, El-Guebaly N. Sociodemographic Factors Associated with Comorbid Major Depressive Episodes and Alcohol Dependence in the General Population. Can J Psychiatry. 2004;49:37-44.

53. Rousseau C, Drapeau A. Premigration exposure to political violence among independent immigrants and its association with emotional distress. J Nerv Ment Dis. 2004;192:852-856.
54. Fry PS, Grover SC. Cognitive Appraisals of Life Stress and Depression in the Elderly: A Cross-Cultural Comparison of Asians and Caucasians. International Journal of Psychology. 1982;17:437-454.

55. Dyal, James A; Chan, Carolina. Stress and distress: A study of Hong Kong Chinese and Euro-Canadian students. Journal of CrossCultural Psychology. 1985;16(4):447-466.

56. Blandford AA, Chappell NL. Subjective well-being among Native and non-Native elderly persons: Do differences exist? Can J Aging. 1990;9(4):386-399.

57. Dion KL, Giordano C. Ethnicity and Sex as Correlates of Depression Symptoms in a Canadian University Sample. Int $\mathrm{J}$ Soc Psychiatry. 1990;36:30-41.

58. Bagley CR. Mental health and social adjustment of elderly Chinese immigrants in Canada. Canada's Mental Health. 1993; Fall:6-10.

59. Dion KL. Ethnolinguistic Correlates of Alexithymia: Toward a Cultural Perspective. J Psychosom Res. 1996;41:531-539.

60. Heine SJ, Lehman DR. Culture, SelfDiscrepancies, and Self-Satisfaction. Pers Soc Psychol Bull. 1999;25:915-925.

61. Rousseau C, Mekki-Berrada A, Moreau S. Trauma and extended separation from family among Latin American and African refugees in Montreal. Psychiatry. 2001;64:40-59.

62. Howard LA, Ahluwalia JS, Lin SK, Sellers EM, Tyndale RF. CYP2E1*1D regulatory polymorphism: association with alcohol and nicotine dependence. Pharmacogenetics. 2003;13:321-328.

63. Tweed RG, White K, Lehman DR. Culture, stress and coping: Internally- and externallytargeted control strategies of European Canadians, East Asian Canadians and Japanese. Journal of Cross-Cultural Psychology. 2004;35:652-668.
64. Khanlou N. Influences on adolescent selfesteem in multicultural Canadian secondary schools. Public Health Nurs. 2004;21:404411.

65. Aubert P, Daigle MS, Daigle JG. Cultural Traits and Immigration: Hostility and Suicidality in Chinese Canadian Students. Trancultural psychiatry. 2004;41: 514-532.

66. Bland RC, Orn H. Schizophrenia: Sociocultural Factors. Can J Psychiatry. 1981; 26:186-188.

67. Seltzer A, Langford A. Forensic psychiatric assessments in the Northwest Territories. Can J Psychiatry. 1984;29:665-668.

68. Borzecki M, Wormith JS, Black WH. An examination of differences between native and non-native psychiatric offenders on the MMPI. Canadian Journal of Behavioural Sciences. 1988;20:287-301.

69. Chandrasena R, Beddage V, Fernando MLD. Suicide among immigrant psychiatric patients in Canada. Br J Psychiatry. 1991;159:707-709.

70. Norton GR, Rockman GE, Malan J, Cox BJ, Hewitt PL. Panic attacks, chemical abuse and suicidal ideation: A comparison of native and non-native Canadians. Alcoholism Treatment Quarterly. 1995;12:33-41.

71. Weekes JR, Morison SJ, Millson WA, Fettig DM. A comparison of Native, Métis, and Caucasian offender profiles on the MCMI. Canadian Journal of Behavioural Science. 1995;27:187-198.

72. Pawliuk N, Grizenko N, Chan-Yip A, Gantous P, Mathew J, Nguyen D. Acculturation style and psychological functioning in children of immigrants. Am J Orthopsychiatry. 1996;66:111-121.

73. Zapf PA, Roesch R, Hart SD. An examination of the relationship of homelessness to mental disorder, criminal behaviour, and health care in a pretrial jail population. Can J Psychiatry. 1996;41:435-440. 
74. Devins GM, Edworthy SM, Aramis Lupus State Models Research Group. Illness intrusiveness explains race-related quality-oflife differences among women with systemic lupus erythematosus. Lupus. 2000;9:534-541.

75. Hodelet N. Psychosis and offending in British Columbia: characteristics of a secure hospital population. Crim Behav Ment Health. 2001;11163-172.

76. Troper H, Weinfeld M. Ethnicity, Politics and Public Policy: Case studies in Canadian Diversity, Toronto, University of Toronto Press, 1999, p. 3-25.

77. Williams DR. Race and Health: Basic Questions, Emerging Directions. Annual Review of Epidemiology. 1997;7:322-333.

78. Isajiw WW. Understanding diversity: Ethnicity and race in the Canadian Context, Toronto, Thompson Educational Publishing Inc., 1999, p. 17-36.

79. Comstock RD, Castillo EM, Lindsay SP. Four-Year Review of the Use of Race and Ethnicity in Epidemiologic and Public Health Research. Am J Epidemiol. 2004; 159:611-619.

80. Trimble JE, Dickson R. " Ethnic Identity ", dans Fisher CB and Lerner RM (dir.), Applied developmental science: An encyclopedia of research, policies, and programs, Thousand Oaks, Sage. (À l'impression)

81. Allahar A. " The social construction of primordial identities ", dans Hier SP and Bolaria BS (dir.), Identity and Belonging: Rethinking race and ethnicity in Canadian society, Toronto, Canadian Scholars' Press Inc., 2006, chapitre 2, p. 31-42.

82. Connor W. Ethnonationalism: The Quest for Understanding, Princeton, Princeton University Press, 1994, p. 89-117.
83. Smith AD. The ethnic origins of nations, New York, Basil Blackwell, 1986, p. 1-20.

84. Van Den Berghe PL. "Ethnicity and the sociobiology debate ", dans J. Rex et D. Mason (dir.), Theories of Race and Ethnic Relations, Cambridge University Press, 1986, p. 246-263.

85. Kaufman SJ. Ethnic fears and ethnic war in Karabagh, Centre for Strategic and International Studies, 1998. URL : http://www. csis.org/ruseura/pronars/working_papers.

86. Cheung YW. Approaches to ethnicity: Clearing roadblocks in the study of ethnicity and substance abuse. International Journal of Addictions. 1993;28(12):1209-1226.

87. Phinney J. «Ethnic identity and acculturation ", dans Chun K, Organista PB, and Marin G (dir.), Acculturation: Advances in theory, measurement, and applied research, Washington (DC), American Psychological Association, 2003, p. 63-81.

88. Tajfel H. Social identity and intergroup relations, Cambridge, Cambridge University Press, 1982, p. 207-240.

89. Weinreich P, Saunderson W (dir.), Analyzing identity: Cross-cultural, societal and clinical contexts, New York, Routledge, 2003, p. 7-110 et p. 115-170.

90. Statistique Canada. Enquête nationale sur la santé de la population, cycle 2. 1996. URL : http://www.statcan.ca/cgi-bin/ $\mathrm{imdb} /$ p2SV_f.pl?Function $=$ getSurvey \&SD $\mathrm{DS}=3225 \&$ lang $=\mathrm{en} \& \mathrm{db}=\mathrm{IMDB} \& \mathrm{dbg}=\mathrm{f} \&$ $\operatorname{adm}=8 \&$ dis $=2$.

91. Statistique Canada. Enquête sur la santé dans les collectivités canadiennes, cycle 1.1. 2000. URL : http://www.statcan.ca/ francais/concepts/health/.
92. Nazroo JY. Genetic, cultural or socioeconomic vulnerability? Explaining ethnic inequalities in health. Sociol Health Illn. 1998;20:710-730.

93. Nazroo JY. The structuring of ethnic inequalities in health: economic position, racial discrimination and racism. Am J Public Health. 2003;93(2):277-84

94. Statistique Canada. Enquête longitudinale national sur les enfants et les jeunes, Cycle 1. 1994. URL : http://www.statcan.ca/cgibin/imdb/p2SV_f.pl? Function = get Survey \& SurvId $=4450 \&$ SurvVer $=0$ \& InstaId $=16044 \&$ InstaVer $=1 \&$ SDDS $=$ 4450 \&lang $=\mathrm{en} \& \mathrm{db}=\mathrm{imdb} \& \mathrm{dbg}=\mathrm{f} \& \mathrm{adm}$ $=8 \&$ dis $=2$.

95. Statistique Canada. Enquête nationale sur le don, le bénévolat et la participation, Cycle 1. 1997. URL : http://www.statcan. ca/cgi-bin/imdb/p2SV_f.pl?Function = get Survey\&SurvId $=4430 \&$ SurvVer $=0 \& I n s t a I$ $\mathrm{d}=16024$ \&InstaVer $=1$ \&SDDS $=4430$ \&lang $=\mathrm{en} \& \mathrm{db}=\mathrm{imdb} \& \mathrm{dbg}=\mathrm{f} \& \mathrm{adm}=8 \& \mathrm{dis}=2$.

96. Statistique Canada. Enquête sociale générale - Victimisation. 1999. URL : http://www. statcan.ca/cgi-bin/imdb/p2SV_f.pl?Functio $\mathrm{n}=$ getSurvey \&SDDS $=4504 \&$ lang $=\mathrm{en} \& \mathrm{db}$ $=I M D B \& d b g=f \& a d m=8 \&$ dis $=2$.

97. LaVeist TA. Beyond Dummy Variables and Sample Selection: What Health Services Researchers Ought to Know about Race as a variable. Health Serv Res. 1994;29:1-16.

98. Yinger JM. Ethnicity: Source of Strength? Source of Conflict?, New York, State University of New York Press, 1994, p. 1-67 et p. 167-198.

99. Stolley PD. Race in Epidemiology. Int J Health Serv. 1999;29:905-909.

100. Chaturvedi N. Ethnicity as an epidemiological determinant-crudely racist or crucially important? Int J Epidemiol. 2001; 30:925-927. 
101. James CE. « Race, ethnicity and cultural identity ", dans Hier SP and Bolaria BS (dir.), Identity and Belonging: Rethinking race and ethnicity in Canadian society, Toronto (Canada), Canadian Scholars' Press Inc., 2006, chapitre 3, p. 43-55.

102. Di Biase S, Bauder H. Immigrants in Ontario: Linking Spatial Settlement Patterns and Labour Force Characteristics, Department of Geography, University of Guelph, 2004. URL : www.uoguelph.ca/geography/ research/ffw/papers/Settlement \%20in \% 20 Ontario_report.pdf.

103. Ali J, Grabb E. Ethnic origin, class origin and educational attainment in Canada: Further evidence on the mosaic thesis. Journal of Canadian Studies. 1998;32: 3-21.

104. Breton R, Roseborough H. Ethnic differences in status. In Blishen BR et al., eds. Canadian Society. Toronto: Macmillan, 1968:683-701.
105. Thompson EN. Résultat des immigrants sur le plan des compétences et rôle du capital humain spécifique à une région d'origine - Septembre 2000, Ottawa, Ressources humaines et Développement social Canada, 2000, $\mathrm{n}^{\circ}$ W-00-8F au catalogue.

106. Wallace M. «Planning Amidst Diversity: The Challenges of Multiculturalism in Urban and Suburban Greater Toronto ", thèse de doctorat, University of Waterloo, 1999.

107. Beshiri, R. Les immigrants au Canada rural : une mise à jour de 2001. Bulletin d'analyse - Régions rurales et petites villes du Canada, vol. 5 (2004), p. 1-27, $n^{\circ}$ 21-006-XIF au catalogue.
108. Schellenberg G. Tendances et conditions dans les régions métropolitaines de recensement : Les immigrants dans les régions métropolitaines de recensement au Canada, Statistique Canada, 2004, $n^{\circ}$ 89-613-MIF au catalogue - $n^{\circ} 003$. URL : http://www.statcan.ca/francais/ research/89-613-MIF/2004003/89-613MIF2004003.pdf.

109. Manitoba and Northern Affairs. Aboriginal People in Manitoba 2000. URL : http:// www.gov.mb.ca/ana/apm2000/index. html.

110. Office of the Registrar General, Inde. Proportion and growth rate of population by religious communities - India : 19612001. URL : http://www.censusindia.net/ religiondata/statement.pdf. 


\section{ANNEXE I \\ Études quantitatives empiriques sur l'ethnicité et la santé mentale menées au Canada (ou à l'aide de données canadiennes) portant sur deux groupes ethniques ou plus}

$\mathrm{N}=$ taille d'échantillon, $\mathrm{A}=$ groupe d'âge des participants, $\mathrm{S}=$ source(s) des données, $\mathrm{D}=$ type de données, $\mathrm{AD}=$ type d'analyse des données, $\mathrm{T}=$ type d'étude, $\mathrm{VD}=$ variable dépendante, $\mathrm{VI}=$ variable indépendante.

\begin{tabular}{|c|c|c|c|c|}
\hline $\begin{array}{l}\text { Auteur(s), année de } \\
\text { publication et région } \\
\text { à l'étude }\end{array}$ & $\begin{array}{l}\text { Échantillon de l'étude (taille de } \\
\text { l'échantillon, source des données, } \\
\text { groupe d'âge inclus et méthodologie) }\end{array}$ & Groupes ethniques inclus & Phénomène étudié & Commentaires \\
\hline \multicolumn{5}{|c|}{ Études fondées sur des enquêtes basées sur une population, sur des enquêtes communautaires de grande envergure ou sur les échantillons de ces enquêtes ( $\mathbf{n}=\mathbf{2 7}$ ) } \\
\hline $\begin{array}{l}\text { 1. Murphy, } 1980 \\
\text { (Canada) }^{29}\end{array}$ & $\begin{array}{l}N=\text { non précisé } \\
A=15 \text { ans et plus } \\
S=\text { Rapports du Dominion Bureau of Statistics } \\
\text { au sujet des premières admissions dans un } \\
\text { hôpital psychiatrique canadien en } 1961 \text { et } \\
\text { recensement de la population de } 1961 . \\
D=\text { Transversales } \\
A D=\text { Ratio standardisé de morbidité } \\
T=\text { Descriptive }\end{array}$ & $\begin{array}{l}\text { Allemands; Néerlandais; } \\
\text { Scandinaves; Nés au Canada }\end{array}$ & $\begin{array}{l}\text { VD : Hospitalisation dans un } \\
\text { établissement psychiatrique }\end{array}$ & $\begin{array}{l}\text { 1. Absence d'une définition claire de } \\
\text { l'ethnicité. } \\
\text { 2. L'ethnicité est établie en fonction du " pays } \\
\text { d'où sont venus les ancêtres paternels de la } \\
\text { personne lorsqu'ils se sont établis au } \\
\text { Canada " [TRADuction], donc il s'agit d'une } \\
\text { conceptualisation en fonction de l'origine } \\
\text { ethnique. } \\
\text { 3. Les groupes ethniques reflètent le modèle } \\
\text { de l'immigration au Canada au cours de la } \\
\text { période visée (c.-à-d. les années 1960). }\end{array}$ \\
\hline $\begin{array}{l}\text { 2. Liban et Smart, } 1982 \\
\text { (Ontario, Canada) }^{30}\end{array}$ & $\begin{array}{l}N=128 \\
A=10 \text { à } 20 \text { ans (c.-à-d. de la } 7^{e} \text { à la } 13^{e} \text { année) } \\
S=1979 \text { Survey of Alcohol and Drug Use } \\
\text { among Ontario students } \\
D=\text { Transversales } \\
A D=\text { Analyses descriptive et du test du chi } \\
\text { carré } \\
T=\text { Descriptive }\end{array}$ & $\begin{array}{l}\text { Autochtones [64]; Allochtones } \\
\text { [64] }\end{array}$ & $\begin{array}{l}\text { VD : Fréquence de l'usage } \\
\text { d'alcool et de drogues et } \\
\text { problèmes liés à cet usage }\end{array}$ & $\begin{array}{l}\text { 1. Absence d'une définition claire de } \\
\text { l'ethnicité. } \\
\text { 2. L'ethnicité est établie en fonction du milieu } \\
\text { culturel, mais la façon dont ce dernier a été } \\
\text { déterminé n'est pas précisée. } \\
\text { 3. Bien que, dans cette étude, le milieu culturel } \\
\text { ait été divisé selon les catégories des } \\
\text { Canadiens anglais, des Canadiens français, } \\
\text { des Asiatiques, des Autochtones et des } \\
\text { autres, les catégories des allochtones ont } \\
\text { été regroupées aux fins de cette étude par } \\
\text { un assortissage. }\end{array}$ \\
\hline $\begin{array}{l}\text { 3. Havens et Chappell, } \\
1983 \text { (Manitoba, } \\
\text { Canada) }^{31}\end{array}$ & $\begin{array}{l}N=3647 \\
A=65 \text { ans et plus } \\
S=\text { Étude longitudinale sur le vieillissement au } \\
\text { Manitoba (1971) } \\
D=\text { Transversales } \\
A D=\text { Analyse de la variance } \\
T=\text { Analytique }\end{array}$ & $\begin{array}{l}\text { Nord-Américains [370]; } \\
\text { Britanniques [1 633] Français } \\
\text { [216]; Polonais/Russes/ } \\
\text { Ukrainiens [685]; Autres } \\
\text { Européens (Allemands, } \\
\text { Norvégiens, Danois, Suédois, } \\
\text { Islandais, Néerlandais, Belges) } \\
\text { [743] }\end{array}$ & $\begin{array}{l}\text { VD : Bien-être perçu, état de } \\
\text { santé perçu, fonctionnement } \\
\text { de la santé mentale }\end{array}$ & $\begin{array}{l}\text { 1. Les groupes ethniques de cette étude ne } \\
\text { sont pas représentatifs de l'ensemble du } \\
\text { Canada, mais plutôt du Manitoba. } \\
\text { 2. Les groupes ethniques sont à l'image du } \\
\text { modèle d'immigration de la province et de } \\
\text { la période visée. } \\
\text { 3. L'origine ethnique est établie grâce à une } \\
\text { question sur l'ethnicité incluse dans cette } \\
\text { enquête. }\end{array}$ \\
\hline $\begin{array}{l}\text { 4. Penning, } 1983 \\
\text { (Canada) }^{32}\end{array}$ & $\begin{array}{l}N=2253 \\
A=30 \text { ans et plus } \\
S=\text { Social Change in Canada Survey (1977) } \\
D=\text { Transversales } \\
A D=\text { Analyse de la variance } \\
T=\text { Analytique }\end{array}$ & $\begin{array}{l}\text { Canadiens [1 720]; Américains } \\
\text { [57]; Britanniques [159]; } \\
\text { Nord-Européens (France, } \\
\text { Allemagne, Autriche, } \\
\text { Scandinavie, Pays-Bas) [96]; } \\
\text { Sud-Européens (Grèce, } \\
\text { Portugal, Espagne, Italie) [65]; } \\
\text { Européens de l'Est (Russie, } \\
\text { Hongrie, Pologne) [96]; Autres } \\
\text { [62] }\end{array}$ & $\begin{array}{l}\text { VD : Bien-être psychologique } \\
\text { perçu }\end{array}$ & $\begin{array}{l}\text { 1. Absence d'une définition claire de } \\
\text { l'ethnicité. } \\
\text { 2. Certaines catégories ethniques ne sont pas } \\
\text { expliquées clairement, comme le groupe } \\
\text { ethnique des " Autres ". } \\
\text { 3. L'origine ethnique est établie selon le pays de } \\
\text { naissance. }\end{array}$ \\
\hline $\begin{array}{l}\text { 5. Tcheng-Laroche et } \\
\text { Prince, } 1983 \text { (Montréal, } \\
\text { Québec, Canada) })^{33}\end{array}$ & $\begin{array}{l}\mathrm{N}=128 \\
\mathrm{~A}=\text { non précisé } \\
\mathrm{S}=\text { Enquête communautaire portant sur un } \\
\text { échantillon représentatif de mères séparées ou } \\
\text { divorcées de Montréal } \\
\mathrm{D}=\text { Transversales } \\
\mathrm{AD}=\text { Test du chi carré, analyse de la variance } \\
\mathrm{T}=\text { Analytique }\end{array}$ & $\begin{array}{l}\text { Francophones [62]; } \\
\text { Anglophones [66] }\end{array}$ & $\begin{array}{l}\text { VD : Stress psychosocial } \\
\text { d'après l'échelle de Langner, } \\
\text { estime de soi selon le QESR et } \\
\text { satisfaction de vivre }\end{array}$ & $\begin{array}{l}\text { 1. Absence d'une définition claire de } \\
\text { l'ethnicité. } \\
\text { 2. Les auteurs de l'étude étaient intéressés à } \\
\text { examiner les " effets sur la culture ». } \\
\text { 3. L'étude porte sur des Canadiennes } \\
\text { françaises et des Canadiennes anglaises de } \\
\text { Montréal. On ne sait pas exactement } \\
\text { comment le statut de Canadienne française } \\
\text { et de Canadienne anglaise a été déterminé. }\end{array}$ \\
\hline $\begin{array}{l}\text { 6. Tonkin, } 1984 \\
\text { (Colombie-Britannique, } \\
\text { Canada) }^{34}\end{array}$ & $\begin{array}{l}\mathrm{N}=122 \\
\mathrm{~A}=<20 \text { ans } \\
\mathrm{S}=\text { Données de l'état civil (et examen de suivi } \\
\text { de tous les décès signalés au bureau du } \\
\text { coroner en chef de la province en 1978-1979) } \\
\mathrm{D}=\text { Transversales } \\
\mathrm{AD}=\text { Analyses descriptive et du test du chi } \\
\text { carré } \\
\mathrm{T}=\text { Descriptive }\end{array}$ & $\begin{array}{l}\text { Autochtones [33]; } \\
\text { Non-Autochtones [89] }\end{array}$ & $\begin{array}{l}\text { VD : Suicides et diagnostics } \\
\text { psychiatriques }\end{array}$ & $\begin{array}{l}\text { 1. Absence d'une définition claire de } \\
\text { l'ethnicité. } \\
\text { 2. L'ethnicité a été utilisée comme covariable, } \\
\text { mais la façon dont elle a été déterminée } \\
\text { n'est pas indiquée, car elle a été soustraite } \\
\text { des dossiers du coroner. } \\
\text { 3. La raison de l'inclusion de l'ethnicité en tant } \\
\text { que covariable n'est pas expliquée. }\end{array}$ \\
\hline
\end{tabular}




\begin{tabular}{|c|c|c|c|c|}
\hline $\begin{array}{l}\text { Auteur(s), année de } \\
\text { publication et région } \\
\text { à l'étude }\end{array}$ & $\begin{array}{l}\text { Échantillon de l'étude (taille de } \\
\text { l'échantillon, source des données, } \\
\text { groupe d'âge inclus et méthodologie) }\end{array}$ & Groupes ethniques inclus & Phénomène étudié & Commentaires \\
\hline $\begin{array}{l}\text { 7. Barnes et coll., } 1988 \\
\text { (Winnipeg, Manitoba, } \\
\text { Canada) }^{35}\end{array}$ & $\begin{array}{l}N=524 \\
A=18 \text { à } 80 \text { ans } \\
S=1983 \text { Winnipeg Area Study } \\
D=\text { Transversales } \\
A D=\text { Analyse de la variance, du test du chi } \\
\text { carré et analyse de classification multiple } \\
T=\text { Exploratoire }\end{array}$ & $\begin{array}{l}\text { Anglais [84]; Européens de } \\
\text { I'Est [62]; Européens de } \\
\text { l'Ouest [82]; Canadiens [193]; } \\
\text { Autres [97] }\end{array}$ & VD : Dépression (CES-D) & $\begin{array}{l}\text { 1. L'ethnicité a été examinée en tant que } \\
\text { variable explicative de la dépression. } \\
\text { 2. Les groupes ethniques sont révélateurs de } \\
\text { la composition ethnique de la région à } \\
\text { l'étude. } \\
\text { 3. L'origine ethnique est établie selon le pays de } \\
\text { naissance. }\end{array}$ \\
\hline $\begin{array}{l}\text { 8. Sack et coll., } 1993 \\
\text { (Canada et É.-U..) }^{36}\end{array}$ & $\begin{array}{l}\mathrm{N}=1115 \\
\mathrm{~A}=7 \text { à } 9 \text { ans (de la } 2^{\mathrm{e}} \text { à la } 4^{\mathrm{e}} \text { année) } \\
\mathrm{S}=\text { Flower of Two Soils Project } \\
\mathrm{D}=\text { Prospectives et longitudinales (suivi de } \\
3 \text { ans) } \\
\mathrm{AD}=\text { Analyses de corrélation, analyse de la } \\
\text { variance et analyse du test du chi carré } \\
\mathrm{T}=\text { Analytique }\end{array}$ & $\begin{array}{l}\text { Enfants des Premières nations } \\
\text { des Plaines (Dakota du Sud, } \\
\text { É.-U.), des Forêts du Nord } \\
\text { (Manitoba, Canada), du Désert } \\
\text { (Nouveau-Mexique, É.-U.) et } \\
\text { des Côtes (Colombie- } \\
\text { Britannique, Canada) } \\
\text { comparés à un échantillon } \\
\text { d'enfants non autochtones } \\
\text { dans chacune des régions }\end{array}$ & $\begin{array}{l}\text { VD : Symptômes dépressifs } \\
\text { décelés grâce à de nouvelles } \\
\text { mesures de psychopathologie } \\
\text { et de santé mentale (les SOS) }\end{array}$ & $\begin{array}{l}\text { 1. Aucune définition explicite de l'ethnicité } \\
\text { n'est fournie. } \\
\text { 2. Les auteurs de l'étude étaient } \\
\text { particulièrement intéressés par les } \\
\text { différences culturelles entre les divers } \\
\text { groupes des Premières nations présents en } \\
\text { Amérique du Nord; il est donc sous-entendu } \\
\text { que l'ethnicité est établie en fonction de la } \\
\text { culture. }\end{array}$ \\
\hline $\begin{array}{l}\text { 9. Walters, } 1993 \\
\text { (Hamilton, Ontario, } \\
\text { Canada) }^{37}\end{array}$ & $\begin{array}{l}N=356 \\
A=21 \text { ans et plus } \\
S=\text { Un échantillon aléatoire stratifié de femmes } \\
\text { de Hamilton } \\
D=\text { Transversales } \\
A D=\text { Test du chi carré } \\
T=\text { Descriptive }\end{array}$ & $\begin{array}{l}\text { Anglophones vs. autres } \\
\text { langues }\end{array}$ & $\begin{array}{l}\text { VD : Stress, anxiété et } \\
\text { dépression }\end{array}$ & $\begin{array}{l}\text { 1. L'origine ethnique est établie selon la } \\
\text { langue maternelle. } \\
\text { 2. Absence d'une définition de l'ethnicité. } \\
\text { 3. La langue est mentionnée comme variable } \\
\text { de substitution possible pour l'ethnicité. }\end{array}$ \\
\hline $\begin{array}{l}\text { 10. Beiser et coll., } 1994 \\
\text { (Vancouver, } \\
\text { Colombie-Britannique, } \\
\text { Canada) })^{38}\end{array}$ & $\begin{array}{l}N=1667 \\
A=\text { ? } \\
S=1348 \text { réfugiés du Projet de réinstallation } \\
\text { des réfugiés et un échantillon aréolaire de } \\
319 \text { résidents de Vancouver appariés aux } \\
\text { réfugiés en fonction de l'âge et du sexe } \\
D=\text { Transversales } \\
A D=\text { Analyse du degré d'appartenance } \\
\text { (méthode GOM : une technique d'agrégation } \\
\text { multidimentionnelle) } \\
T=\text { Analytique }\end{array}$ & $\begin{array}{l}\text { Asiatiques du Sud-Est [1 348]; } \\
\text { Résidents Canadiens [319]; } \\
\text { Groupe des Asiatiques du } \\
\text { Sud-Est désagrégé : Chinois } \\
\text { [755]; Vietnamiens/Laotiens } \\
\text { [593] }\end{array}$ & $\begin{array}{l}\text { VD : Troubles psychiatriques, } \\
\text { dont la dépression, l'anxiété } \\
\text { et la somatisation, selon la } \\
\text { CES-D, le questionnaire DIS et } \\
\text { le questionnaire de santé du } \\
\text { Sénégal }\end{array}$ & $\begin{array}{l}\text { 1. Aucune définition explicite de l'ethnicité } \\
\text { n'est fournie. } \\
\text { 2. Les chercheurs étaient intéressés par } \\
\text { "l'expression psychopathologique parmi } \\
\text { différents groupes ethnoculturels " } \\
\text { [TRADuction]; l'ethnicité repose donc sur la } \\
\text { culture. } \\
\text { 3. L'ethnicité est établie en fonction de } \\
\text { l'endroit d'où la personne a émigré. }\end{array}$ \\
\hline $\begin{array}{l}\text { 11. Beiser et coll., } 1998 \\
\text { (Canada et É.-U.) }^{39}\end{array}$ & $\begin{array}{l}\mathrm{N}=1708 \\
\mathrm{~A}=7 \text { à } 9 \text { ans (de la } 2^{\mathrm{e}} \text { à la } 4^{\mathrm{e}} \text { année) } \\
\mathrm{S}=\text { Flower of Two Soils Project } \\
\mathrm{D}=\text { Prospectives et longitudinales } \\
\mathrm{AD}=\text { Analyse factorielle des composantes } \\
\text { principales sur la mesure de la } \\
\text { psychopathologie, analyses de corrélation, } \\
\text { analyse de la variance, analyses du test du chi } \\
\text { carré } \\
\mathrm{T}=\text { Analytique }\end{array}$ & $\begin{array}{l}\text { Enfants des Premières nations } \\
\text { des Plaines (Dakota du Sud, } \\
\text { É.-U.), des Forêts du Nord } \\
\text { (Manitoba, Canada), du Désert } \\
\text { (Nouveau-Mexique, É.-U.) et } \\
\text { des Côtes (Colombie- } \\
\text { Britannique, Canada) [1 251] } \\
\text { comparés à un échantillon } \\
\text { d'enfants non autochtones } \\
\text { dans chacune des régions } \\
\text { [457] }\end{array}$ & $\begin{array}{l}\text { VD : Symptômes dépressifs } \\
\text { décelés grâce à de nouvelles } \\
\text { mesures de psychopathologie } \\
\text { et de santé mentale (les SOS) }\end{array}$ & $\begin{array}{l}\text { 1. Aucune définition explicite de l'ethnicité } \\
\text { n'est fournie. } \\
\text { 2. Les auteurs de l'étude étaient } \\
\text { particulièrement intéressés par les } \\
\text { différences culturelles entre les divers } \\
\text { groupes des Premières nations présents en } \\
\text { Amérique du Nord. }\end{array}$ \\
\hline $\begin{array}{l}\text { 12. De Wit et coll., } 1999 \\
\text { (Ontario, Canada) }^{40}\end{array}$ & $\begin{array}{l}\mathrm{N}=4531 \\
\mathrm{~A}=19 \text { ans et plus } \\
\mathrm{S}=\text { La Native Ontario Community Survey et le } \\
\text { supplément sur la santé mentale de l'Enquête } \\
\text { sur la santé en Ontario. } \\
\mathrm{D}=\text { Transversales (mais les données sur l'âge à } \\
\text { la première consommation ont servi à étudier } \\
\text { l'incidence) } \\
\mathrm{AD}=\text { Analyse descriptive, analyse du test du } \\
\text { chi carré et analyse de survie } \\
\mathrm{T}=\text { Analytique }\end{array}$ & $\begin{array}{l}\text { Autochtones [876]; } \\
\text { Non-Autochtones [3 655] }\end{array}$ & $\begin{array}{l}\text { VD : Usage d'alcool et de } \\
\text { drogues et date de la } \\
\text { première consommation }\end{array}$ & $\begin{array}{l}\text { 1. Aucune définition explicite de l'ethnicité } \\
\text { n'est fournie. } \\
\text { 2. Les auteurs de cette étude étaient } \\
\text { particulièrement intéressés par les } \\
\text { différences culturelles entre les Autochtones } \\
\text { et les non-Autochtones au Canada. }\end{array}$ \\
\hline $\begin{array}{l}\text { 13. De Wit et Beneteau, } \\
1999 \text { (Ontario, } \\
\text { Canada) }^{41}\end{array}$ & $\begin{array}{l}\mathrm{N}=5150 \\
\mathrm{~A}=16 \text { ans et plus } \\
\mathrm{S}=\text { Enquête sur la santé en Ontario de } 1990 \\
\mathrm{D}=\text { Transversales, mais l'âge à la première } \\
\text { consommation a permis d'effectuer des } \\
\text { analyses de survie } \\
\mathrm{AD}=\text { Test du chi carré, analyse de survie et } \\
\text { analyse de régression logistique } \\
\mathrm{T}=\text { Analytique }\end{array}$ & 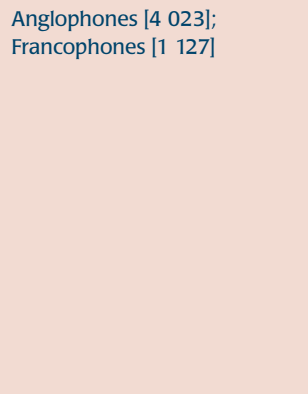 & $\begin{array}{l}\text { VD : Consommation d'alcool } \\
\text { (c.-à-d. fréquence et volume); } \\
\text { problèmes liés à l'alcool } \\
\text { (c.-̀̀-d. conduite en état } \\
\text { d'ébriété, conflits familiaux, } \\
\text { conflits au travail, recherche } \\
\text { d'aide pour régler le problème } \\
\text { de consommation d'alcool, } \\
\text { hospitalisation en raison } \\
\text { d'une consommation d'alcool } \\
\text { ou arrestation pour avoir eu } \\
\text { un mauvais comportement } \\
\text { attribuable à la } \\
\text { consommation d'alcool) }\end{array}$ & $\begin{array}{l}\text { 1. Aucune définition explicite de l'ethnicité } \\
\text { n'est fournie. } \\
\text { 2. L'ethnicité est établie en fonction d'une } \\
\text { combinaison de l'identité ethnique et de la } \\
\text { langue principale utilisée à la maison. }\end{array}$ \\
\hline
\end{tabular}




\begin{tabular}{|c|c|c|c|c|}
\hline $\begin{array}{l}\text { Auteur(s), année de } \\
\text { publication et région } \\
\text { à l'étude }\end{array}$ & $\begin{array}{l}\text { Échantillon de l'étude (taille de } \\
\text { l'échantillon, source des données, } \\
\text { groupe d'âge inclus et méthodologie) }\end{array}$ & Groupes ethniques inclus & Phénomène étudié & Commentaires \\
\hline $\begin{array}{l}\text { 14. De Wit et Beneteau, } \\
\text { 1999 (Ontario, } \\
\text { Canada) }^{42}\end{array}$ & $\begin{array}{l}N=5150 \\
A=16 \text { ans et plus } \\
S=\text { Enquête sur la santé en Ontario de } 1990 \\
D=\text { Transversales, mais l'âge à la première } \\
\text { consommation a permis d'effectuer des } \\
\text { analyses de survie } \\
A D=\text { Analyse du test du chi carré, analyse de } \\
\text { survie et analyse de régression logistique } \\
T=\text { Analytique }\end{array}$ & $\begin{array}{l}\text { Anglophones [l } 023] \\
\text { Francophones [l } 127]\end{array}$ & $\begin{array}{l}\text { VD : Consommation de tabac } \\
\text { quotidienne (c.à-d. fréquence } \\
\text { et volume) }\end{array}$ & $\begin{array}{l}\text { 1. Aucune définition explicite de l'ethnicité } \\
\text { n'est fournie. } \\
\text { 2. L'ethnicité est établie en fonction d'une } \\
\text { combinaison de l'identité ethnique et de la } \\
\text { langue principale utilisée à la maison. }\end{array}$ \\
\hline $\begin{array}{l}\text { 15. Feldman et coll., } \\
1999 \text { (Toronto, Ontario, } \\
\text { Canada) }^{43}\end{array}$ & $\begin{array}{l}\mathrm{N}=1236 \\
\mathrm{~A}=\text { Élèves de la } 9^{\mathrm{e}} \text { à la } 13^{\mathrm{e}} \text { année } \\
\mathrm{S}=\text { Enquête portant sur les élèves de la } 9^{\mathrm{e}} \text { à la } \\
13^{\mathrm{e}} \text { année de l'arrondissement de East York, à } \\
\text { Toronto, menée en } 1994 \\
\mathrm{D}=\text { Transversales } \\
\mathrm{AD}=\text { Analyses descriptives, analyses stratifiées } \\
\text { et analyses de régression logistique multiple } \\
\mathrm{T}=\text { Analytique }\end{array}$ & $\begin{array}{l}\text { Canadiens [379]; Européens } \\
\text { [277]; Asiatiques [314]; Autres } \\
\text { [140]; Non précisé [126] }\end{array}$ & $\begin{array}{l}\text { VD : Croyances et } \\
\text { comportements relatifs à } \\
\text { l'usage d'alcool }\end{array}$ & $\begin{array}{l}\text { 1. Aucune définition de l'ethnicité n'est } \\
\text { fournie. } \\
\text { 2. La question * Veuillez inscrire le terme qui } \\
\text { décrit le mieux le caractère ethnique de } \\
\text { votre milieu de vie quotidien * [Traduction] a } \\
\text { été utilisée pour déterminer l'ethnicité, ce } \\
\text { qui indique une conceptualisation en } \\
\text { fonction de l'identité ethnique. }\end{array}$ \\
\hline $\begin{array}{l}\text { 16. Beiser et coll., } 2000 \\
\text { (Canada et É.-U.) }^{44}\end{array}$ & $\begin{array}{l}\mathrm{N}=2044 \\
\mathrm{~A}=7 \text { à } 9 \text { ans (de la } 2^{\mathrm{e}} \text { à la } 4^{\mathrm{e}} \text { année) } \\
\mathrm{S}=\text { Le Flower of Two Soils Project et la School } \\
\text { Option for Native Children Study } \\
\mathrm{D}=\text { Prospectives et longitudinales } \\
\mathrm{AD}=\text { Analyse factorielle des composantes } \\
\text { principales sur la mesure de la } \\
\text { psychopathologie, analyses de corrélation, } \\
\text { analyse de la variance, analyses du test du chi } \\
\text { carré } \\
\mathrm{T}=\text { Analytique }\end{array}$ & $\begin{array}{l}\text { Enfants des Premières nations } \\
\text { des Plaines (Dakota du Sud, } \\
\text { É.-U.), des Forêts du Nord } \\
\text { (Manitoba, Canada), du Désert } \\
\text { (Nouveau-Mexique, É.-U.) et } \\
\text { des Côtes (Colombie- } \\
\text { Britannique, Canada) [1 555]; } \\
\text { comparés à un échantillon } \\
\text { d'enfants non autochtones } \\
\text { dans chacune des régions } \\
\text { [489] }\end{array}$ & $\begin{array}{l}\text { VD : Trouble d'hyperactivité } \\
\text { avec déficit de l'attention } \\
\text { (THADA), selon l'échelle de } \\
\text { symptômes du DSM, mais } \\
\text { certains items ont été mesurés } \\
\text { selon les échelles TIF, CAP et } \\
\text { SOS, dont certains items sont } \\
\text { tirés du CBCL, des CPTRS et } \\
\text { du DIS for Children }\end{array}$ & $\begin{array}{l}\text { 1. Aucune définition explicite de l'ethnicité } \\
\text { n'est fournie. } \\
\text { 2. Les auteurs de l'étude étaient } \\
\text { particulièrement intéressés par les } \\
\text { différences culturelles entre les divers } \\
\text { groupes des Premières nations présents en } \\
\text { Amérique du Nord. Il est donc sous-entendu } \\
\text { que l'ethnicité est établie en fonction de la } \\
\text { culture. }\end{array}$ \\
\hline $\begin{array}{l}\text { 17. Lavallée et } \\
\text { Bourgault, } 2000 \\
\text { (Canada) }^{45}\end{array}$ & $\begin{array}{l}N=27130 \text { femmes } \\
A=15 \text { ans et plus } \\
S=\text { Enquête sur la santé des Cris de 1991, Étude } \\
\text { sur la santé des Inuits de } 1992 \text { et Enquête } \\
\text { sociale et de santé de 1992-1993 } \\
D=\text { Transversales } \\
A D=\text { Analyses de distribution de fréquence } \\
\text { pondérée, du test du chi carré et analyse de la } \\
\text { variance } \\
T=\text { Descriptive }\end{array}$ & $\begin{array}{l}\text { Cris [1 999]; Inuits [1 597]; } \\
\text { Québécoises du Sud de la } \\
\text { province [23 564] }\end{array}$ & $\begin{array}{l}\text { VD : Consommation d'alcool, } \\
\text { usage illicite de drogues, } \\
\text { détresse psychologique, } \\
\text { pensées suicidaires } \\
\text { permanentes }\end{array}$ & $\begin{array}{l}\text { 1. Les chercheuses étaient intéressées par la } \\
\text { santé mentale des femmes Cris, Inuits et du } \\
\text { Sud du Québec, donc l'ethnicité n'est pas } \\
\text { mentionnée, ni définie. } \\
\text { 2. Le groupe " Québécoises du Sud de la } \\
\text { province " est probablement constitué de } \\
\text { multiples groupes ethniques. }\end{array}$ \\
\hline 18. Ali, 2002 (Canada) $)^{5}$ & $\begin{array}{l}\mathrm{N}=92379 \\
\mathrm{~A}=15 \text { à } 75 \text { ans } \\
\mathrm{S}=\text { Enquête sur la santé dans les collectivités } \\
\text { canadiennes, Cycle } 1.1 \\
\mathrm{D}=\text { Transversales } \\
\mathrm{AD}=\text { Analyse de régression logistique multiple } \\
\mathrm{T}=\text { Analytique }\end{array}$ & $\begin{array}{l}\text { É.-U./Mexique [952]; } \\
\text { Amérique du Sud, Amérique } \\
\text { centrale, Caraïbes [2 273]; } \\
\text { Europe [7 749]; Afrique [1 } \\
\text { 139]; Asie [6 314] }\end{array}$ & $\begin{array}{l}\text { VD : Dépression et } \\
\text { dépendance à l'alcool }\end{array}$ & $\begin{array}{l}\text { 1. L'origine ethnique est établie selon la région } \\
\text { de naissance. } \\
\text { 2. Bien que les immigrants soient divisés } \\
\text { d'après la région d'où ils ont migré, le } \\
\text { groupe des personnes nées au Canada n'a } \\
\text { pas été divisé, ce qui limite les } \\
\text { comparaisons en fonction de l'ethnicité. }\end{array}$ \\
\hline $\begin{array}{l}\text { 19. Beiser et coll., } 2002 \\
\text { (Canada) }^{46}\end{array}$ & $\begin{array}{l}\mathrm{N}=13349 \\
\mathrm{~A}=4 \text { à } 11 \text { ans } \\
\mathrm{S}=\text { Enquête longitudinale nationale sur les } \\
\text { enfants et les jeunes (ELNEJ 1994-1995) } \\
D=\text { Transversales } \\
\mathrm{AD}=\text { Analyses de régression logistique multiple } \\
\mathrm{T}=\text { Analytique }\end{array}$ & $\begin{array}{l}\text { Immigrants [684]; Nés au } \\
\text { Canada de parents } \\
\text { immigrants [2 573]; Nés au } \\
\text { Canada de parents nés au } \\
\text { Canada [10 092]. } \\
\text { L'ethnicité a ensuite été } \\
\text { examinée (Asiatiques, Noirs, } \\
\text { autres, Blancs/ Européens). }\end{array}$ & $\begin{array}{l}\text { VD : Problèmes émotionnels } \\
\text { et comportementaux }\end{array}$ & $\begin{array}{l}\text { 1. L'ethnicité a été examinée en tant que } \\
\text { covariable. } \\
\text { 2. L'origine ethnique est établie d'après une } \\
\text { question sur l'ethnicité. } \\
\text { 3. La race ou l'ethnicité des membres de la } \\
\text { catégorie des " autres " n'est pas clairement } \\
\text { définie. }\end{array}$ \\
\hline 20. Ma, 2002 (Canada) $)^{47}$ & $\begin{array}{l}N=2304 \\
A=7 \text { à } 11 \text { ans } \\
S=\text { ELNEJ 1994-1995 } \\
D=\text { Transversales } \\
A D=\text { Analyse factorielle et modélisation } \\
\text { hiérarchique } \\
T=\text { Analytique }\end{array}$ & $\begin{array}{l}\text { Enfants immigrants [182]; } \\
\text { Enfants non immigrants [2 } \\
122] \text {. L'ethnicité a ensuite été } \\
\text { examinée en tant que } \\
\text { covariable (É.-U., Europe, Asie, } \\
\text { autres régions). }\end{array}$ & $\begin{array}{l}\text { VD : Trouble des conduites, } \\
\text { agression indirecte, infractions } \\
\text { contre les biens, } \\
\text { comportement hyperactif, } \\
\text { trouble de comportement } \\
\text { social, perturbation affective } \\
\text { et indice agrégatif de trouble } \\
\text { comportemental ou affectif }\end{array}$ & $\begin{array}{l}\text { 1. L'origine ethnique est établie selon la région } \\
\text { de naissance. } \\
\text { 2. L'ethnicité a été examinée en tant que } \\
\text { covariable. } \\
\text { 3. Aucune définition de l'ethnicité n'est } \\
\text { fournie. }\end{array}$ \\
\hline
\end{tabular}




\begin{tabular}{|c|c|c|c|c|}
\hline $\begin{array}{l}\text { Auteur(s), année de } \\
\text { publication et région } \\
\text { à l'étude }\end{array}$ & $\begin{array}{l}\text { Échantillon de l'étude (taille de } \\
\text { l'échantillon, source des données, } \\
\text { groupe d'âge inclus et méthodologie) }\end{array}$ & Groupes ethniques inclus & Phénomène étudié & Commentaires \\
\hline $\begin{array}{l}\text { 21. Mata, } 2002 \\
\text { (Canada) }^{48}\end{array}$ & $\begin{array}{l}N=17109 \\
A=15 \text { ans et plus } \\
S=\text { Enquête nationale sur le don, le bénévolat } \\
\text { et la participation } \\
D=\text { Transversales } \\
A D=\text { Analyses de régression multiple } \\
T=\text { Analytique }\end{array}$ & $\begin{array}{l}19 \text { catégories ethniques } \\
\text { simples/multiples (Canadiens, } \\
\text { Français, Britanniques, } \\
\text { Allemands, Ukrainiens, } \\
\text { Polonais, Italiens/Portugais, } \\
\text { Néerlandais, Chinois, Noirs/ } \\
\text { Sud-Asiatiques, Autochtones, } \\
\text { Canadiens et Français, } \\
\text { Canadiens et Britanniques, } \\
\text { Canadiens et autres, } \\
\text { Britanniques et Français, } \\
\text { Français et autres, Européens, } \\
\text { reste des catégories simples, } \\
\text { reste des catégories multiples) }\end{array}$ & $\begin{array}{l}\text { VD : Satisfaction de vivre } \\
\text { (échelle à } 4 \text { points de Likert) }\end{array}$ & $\begin{array}{l}\text { 1. L'origine ethnique est établie grâce à une } \\
\text { question sur l'ethnicité incluse dans cette } \\
\text { enquête. } \\
\text { 2. La répartition en groupes ethniques } \\
\text { mutuellement exclusifs est motivée en } \\
\text { partie par des problèmes de taille } \\
\text { d'échantillon. } \\
\text { 3. La raison du groupement de certaines } \\
\text { ethnies n'est pas claire. } \\
\text { 4. Aucune définition de l'ethnicité n'est } \\
\text { fournie. }\end{array}$ \\
\hline $\begin{array}{l}\text { 22. Wu et Hart, } 2002 \\
\text { (Canada) }^{49}\end{array}$ & $\begin{array}{l}N=3009 \text { immigrants âgés } \\
A=65 \text { ans et plus } \\
S=\text { Enquête nationale sur la santé de la } \\
\text { population (ENSP, 1996) } \\
D=\text { Volet transversal } \\
A D=\text { Analyses descriptives et de régression } \\
\text { linéaire multiple } \\
T=\text { Analytique }\end{array}$ & $\begin{array}{l}\text { Chinois/Sud-Asiatiques [274]; } \\
\text { Autres [2 735] }\end{array}$ & $\begin{array}{l}\text { VD : Problèmes émotionnels } \\
\text { et détresse psychologique } \\
\text { (CIDI) }\end{array}$ & $\begin{array}{l}\text { 1. L'ethnicité est établie grâce à une question } \\
\text { sur l'origine ethnique incluse dans cette } \\
\text { enquête. } \\
\text { 2. La combinaison des Chinois et de } \\
\text { l'ensemble des Sud-Asiatiques indique que } \\
\text { l'origine ethnique est établie en fonction } \\
\text { d'une grande région géoculturelle. }\end{array}$ \\
\hline $\begin{array}{l}\text { 23. Wu et coll., } 2003 \\
(\text { Canada })^{2}\end{array}$ & $\begin{array}{l}N=70538 \\
A=12 \text { ans et plus } \\
S=E N S P, 1996 \\
D=\text { Volet transversal } \\
A D=\text { Analyses descriptive et de régression } \\
\text { linéaire multiple } \\
T=\text { Analytique }\end{array}$ & $\begin{array}{l}\text { Asiatiques de I'Est et du } \\
\text { Sud-Est [624]; Chinois [800]; } \\
\text { Sud-Asiatiques [809]; } \\
\text { Autochtones [975]; Noirs } \\
\text { [788]; Arabes et Asiatiques de } \\
\text { l'Ouest [325]; } \\
\text { Latino-Américains [176]; Juifs } \\
\text { [197]; Français [5 580]; } \\
\text { Anglais [9 281]; « autres " } \\
\text { Blancs [50 294]; races mixtes } \\
\text { [689] }\end{array}$ & VD : Dépression (CIDI) & $\begin{array}{l}\text { 1. L'ethnicité est établie grâce à une question } \\
\text { sur l'origine ethnique incluse dans cette } \\
\text { enquête, et elle a été utilisée en } \\
\text { combinaison avec la race pour créer des } \\
\text { groupes ethnoraciaux. } \\
\text { 2. Les définitions de la race et de l'ethnicité } \\
\text { sont fournies a priori. }\end{array}$ \\
\hline $\begin{array}{l}\text { 24. Blackstock et coll., } \\
2004 \text { (Canada) }^{50}\end{array}$ & $\begin{array}{l}\mathrm{N}=3159 \\
\mathrm{~A}=\text { Enfance (groupe d'âge non précisé) } \\
\mathrm{S}=\text { Étude canadienne sur l'incidence des } \\
\text { signalements de cas de violence et de } \\
\text { négligence envers les enfants de } 1998 \text { ( } \mathrm{ECl}-98) \\
\mathrm{D}=\text { Transversales } \\
\mathrm{AD}=\text { Analyse du test du chi carré et analyse de } \\
\text { la variance } \\
\mathrm{T}=\text { Descriptive }\end{array}$ & $\begin{array}{l}\text { Autochtones [614]; Blancs [2 } \\
\text { 114]; Autres minorités visibles } \\
\text { [431] }\end{array}$ & $\begin{array}{l}\text { VD : Fréquence du mauvais } \\
\text { traitement subi par les enfants } \\
\text { (abus physique et sexuel et } \\
\text { négligence); problèmes } \\
\text { psychosociaux. }\end{array}$ & $\begin{array}{l}\text { 1. Aucune définition explicite de l'ethnicité } \\
\text { n'est fournie. } \\
\text { 2. La classification ethnoraciale a été } \\
\text { déterminée d'après le statut ethnoracial de } \\
\text { l'un ou des deux parents biologiques. } \\
\text { 3. L'ethnicité est conceptualisée en fonction de } \\
\text { l'origine ethnique. }\end{array}$ \\
\hline $\begin{array}{l}\text { 25. Cohen et Maclean, } \\
2004 \text { (Canada) }^{51}\end{array}$ & $\begin{array}{l}\mathrm{N}=26000 \\
\mathrm{~A}=15 \text { ans et plus } \\
\mathrm{S}=\text { Tous les participants de sexe féminin à } \\
\text { l'Enquête sociale générale de } 1999 \\
\mathrm{D}=\text { Transversales } \\
\mathrm{AD}=\text { Test } \mathrm{z} \text { ayant comme seuil de signification } \\
p<0,05 \\
\mathrm{~T}=\text { Descriptive }\end{array}$ & $\begin{array}{l}\text { Minorité visible par rapport à } \\
\text { minorité non visible; } \\
\text { Autochtones par rapport à } \\
\text { non-Autochtones }\end{array}$ & $\begin{array}{l}\text { VD : Abus physique, sexuel, } \\
\text { financier ou émotionnel; prise } \\
\text { de médicaments contre } \\
\text { l'anxiété, la dépression ou } \\
\text { l'insomnie chez les personnes } \\
\text { abusées. }\end{array}$ & $\begin{array}{l}\text { 1. L'ethnicité est établie en fonction du pays } \\
\text { de naissance. } \\
\text { 2. Aucune définition de minorité visible n'est } \\
\text { fournie. }\end{array}$ \\
\hline $\begin{array}{l}\text { 26. Wang et El-Guebaly, } \\
2004 \text { (Canada) }^{52}\end{array}$ & $\begin{array}{l}N=72940 \\
A=12 \text { ans et plus } \\
S=\text { ENSP (1996-1997) } \\
D=\text { Transversales } \\
A D=\text { Analyses de régression logistique multiple } \\
T=\text { Analytique }\end{array}$ & $\begin{array}{l}\text { Blancs [67 802]; Non-Blancs } \\
{\left[\begin{array}{ll}5 & 138\end{array}\right]}\end{array}$ & $\begin{array}{l}\text { VD : Épisode de dépression } \\
\text { majeure, dépendance à } \\
\text { l'alcool et utilisation de } \\
\text { services en matière de santé } \\
\text { mentale }\end{array}$ & $\begin{array}{l}\text { 1. L'ethnicité a été examinée en tant que } \\
\text { covariable. } \\
\text { 2. Aucune description n'est faite des groupes } \\
\text { inclus dans la catégorie des non-Blancs. } \\
\text { 3. La population des non-Blancs englobait } \\
\text { vraisemblablement les Autochtones, un } \\
\text { groupe extrêmement susceptible de vivre } \\
\text { des épisodes de dépression majeure et une } \\
\text { dépendance à l'alcool, ce qui explique les } \\
\text { risques élevés observés chez les } \\
\text { non-immigrants et non-Blancs. } \\
\text { 4. La catégorisation est en fonction de la race } \\
\text { seulement, malgré l'emploi du terme } \\
\text { " ethnicité ". }\end{array}$ \\
\hline
\end{tabular}




\begin{tabular}{|c|c|c|c|c|}
\hline $\begin{array}{l}\text { Auteur(s), année de } \\
\text { publication et région } \\
\text { à l'étude }\end{array}$ & $\begin{array}{l}\text { Échantillon de l'étude (taille de } \\
\text { l'échantillon, source des données, } \\
\text { groupe d'âge inclus et méthodologie) }\end{array}$ & Groupes ethniques inclus & Phénomène étudié & Commentaires \\
\hline $\begin{array}{l}\text { 27. Rousseau et } \\
\text { Drapeau, } 2004 \\
\text { (Montréal, Québec, } \\
\text { Canada) }\end{array}$ & $\begin{array}{l}\mathrm{N}=1871 \\
\mathrm{~A}=15 \text { à } 87 \text { ans } \\
\mathrm{S}=\text { Étude auprès des communautés culturelles } \\
\text { du Québec (enquête portant sur les immigrants } \\
\text { récents qui vivent dans la région métropolitaine } \\
\text { de Montréal et qui sont arrivés au Canada } \\
\text { entre } 1988 \text { et } 1997 \text { ) } \\
D=\text { Transversales } \\
A D=\text { Test du chi carré, test } t \text {, analyse de la } \\
\text { variance } \\
T=\text { Descriptive }\end{array}$ & $\begin{array}{l}\text { Chinois; Arabes; Haïtiens; } \\
\text { Hispaniques } \\
\text { Note : il est indiqué dans } \\
\text { l'article qu'un nombre égal de } \\
\text { participants de chacun des } \\
\text { groupes ethniques a été } \\
\text { sélectionné dans le registre } \\
\text { pour représenter la } \\
\text { population cible [n }=750 * 4 \\
=3000] \text {, mais la population } \\
\text { admissible était de } 1871 \\
\text { personnes, sans division des } \\
\text { nombres dans chacun des } \\
\text { groupes ethniques. }\end{array}$ & $\begin{array}{l}\text { VD : Trouble émotionnel } \\
\text { (c.-à-d. dépression et anxiété) } \\
\text { selon le SCL-25, établi grâce à } \\
\text { la liste de contrôle des } \\
\text { symptômes de Hopkins }\end{array}$ & $\begin{array}{l}\text { 1. Aucune définition explicite de l'ethnicité } \\
\text { n'est fournie. } \\
\text { 2. Les chercheuses étaient intéressées par les } \\
\text { immigrants récents de la région de } \\
\text { Montréal qui sont nés dans l'une des quatre } \\
\text { régions géoculturelles suivantes : la Chine, } \\
\text { Hong Kong, Taiwan et Macao [Chinois], } \\
\text { Haïti [Haïtiens], l'Afrique du Nord et le } \\
\text { Moyen-Orient [Arabes] et l'Amérique latine } \\
\text { [Hispaniques]. Par conséquent, l'ethnicité est } \\
\text { conceptualisée en fonction de la région de } \\
\text { naissance. }\end{array}$ \\
\hline \multicolumn{5}{|c|}{ Études de moindre envergure impliquant une collecte de données brutes - Échantillon non clinique $(n=12)$} \\
\hline $\begin{array}{l}\text { 28. Fry et Grover, } 1982 \\
\text { (Canada et É.-U.. }^{54}\end{array}$ & $\begin{array}{l}\mathrm{N}=320 \\
\mathrm{~A}=65 \text { à } 80 \text { ans } \\
\mathrm{S}=\text { Échantillon aléatoire obtenu dans les clubs } \\
\text { professionnels, les associations } \\
\text { communautaires, les centres récréatifs pour les } \\
\text { personnes âgées, les bureaux d'aide sociale et } \\
\text { les maisons privées. } \\
\mathrm{D}=\text { Transversales } \\
\mathrm{AD}=\text { Analyse de la variance } \\
\mathrm{T}=\text { Analytique }\end{array}$ & $\begin{array}{l}\text { Indo-Asiatiques [160]; } \\
\text { Caucasiens [160] }\end{array}$ & $\begin{array}{l}\text { VD : Dépression (selon le BDI), } \\
\text { stress quotidien [selon le Life } \\
\text { Event Inventory], évaluation } \\
\text { cognitive et source de } \\
\text { détermination }\end{array}$ & $\begin{array}{l}\text { 1. Aucune définition de l'ethnicité n'est } \\
\text { fournie. } \\
\text { 2. La façon dont l'ethnicité a été déterminée } \\
\text { n'est pas claire. } \\
\text { 3. La catégorisation semble être en fonction } \\
\text { de la race seulement. }\end{array}$ \\
\hline $\begin{array}{l}\text { 29. Dyal et Chan, } 1985 \\
\text { (Waterloo, Ontario, } \\
\text { Canada) }^{55}\end{array}$ & $\begin{array}{l}\mathrm{N}=251 \\
\mathrm{~A}=17 \text { à } 29 \text { ans } \\
\mathrm{S}=\text { Échantillons de commodité (c.-̀̀-d. étudiants } \\
\text { inscrits à un cours menant à un diplôme de } \\
\text { premier cycle et quelques volontaires) } \\
\mathrm{D}=\text { Transversales } \\
\mathrm{AD}=\text { Analyse de la variance et analyse de la } \\
\text { covariance } \\
\mathrm{T}=\text { Analytique }\end{array}$ & $\begin{array}{l}\text { Euro-Canadiens [112]; Chinois } \\
\text { de Hong Kong [100]; } \\
\text { Immigrants chinois au Canada } \\
\text { [39] }\end{array}$ & $\begin{array}{l}\text { VD : Événements stressants } \\
\text { de la vie selon la Problems } \\
\text { with Living Adjustment scale } \\
\text { (créée pour cette étude); } \\
\text { détresse selon la DSS, fondée } \\
\text { sur l'échelle à } 22 \text { points de } \\
\text { Langer sur l'altération du } \\
\text { fonctionnement; } 12 \text { points de } \\
\text { la DDS; inquiétude selon } \\
\text { l'échelle d'inquiétude SEAS. }\end{array}$ & $\begin{array}{l}\text { 1. Aucune définition explicite de l'ethnicité } \\
\text { n'est fournie. } \\
\text { 2. Il est sous-entendu que l'ethnicité est } \\
\text { établie selon la culture, étant donné que les } \\
\text { auteurs de l'étude sont intéressés par les } \\
\text { différences entre les cultures. } \\
\text { 3. On ne sait pas exactement si le groupe des } \\
\text { Euro-Canadiens s'identifie vraiment comme } \\
\text { tel ou si cette catégorisation repose sur les } \\
\text { observations des chercheurs. }\end{array}$ \\
\hline $\begin{array}{l}\text { 30. Blandford et } \\
\text { Chappell, } 1990 \\
\text { (Winnipeg, Manitoba, }_{\text {Canada) }}^{56}\end{array}$ & $\begin{array}{l}\mathrm{N}=390 \\
\mathrm{~A}=50 \text { ans et plus } \\
\mathrm{S}=\text { Enquête sur les Autochtones à Winnipeg de } \\
1981 \\
\mathrm{D}=\text { Transversales } \\
\mathrm{AD}=\text { Analyses du test du chi carré et de } \\
\text { régression logistique } \\
\mathrm{T}=\text { Analytique }\end{array}$ & $\begin{array}{l}\text { Autochtones [193]; } \\
\text { Non-Autochtones [197] }\end{array}$ & $\begin{array}{l}\text { VD : Satisfaction de vivre; } \\
\text { solitude selon l'échelle de } \\
\text { mesure de la solitude de } \\
\text { I'UCLA }\end{array}$ & $\begin{array}{l}\text { 1. L'ethnicité n'est pas définie de façon précise. } \\
\text { 2. L'ethnicité est conceptualisée en fonction de } \\
\text { l'identité ethnique, mais il n'y a aucune } \\
\text { indication de la façon dont l'identité } \\
\text { ethnique a été déterminée. }\end{array}$ \\
\hline $\begin{array}{l}\text { 31. Dion et Giordano, } \\
1990 \text { (Toronto, Ontario, } \\
\text { Canada) }^{57}\end{array}$ & $\begin{array}{l}\mathrm{N}=352 \\
\mathrm{~A}=\text { Étudiants de première année d'université } \\
\text { - âge moyen de } 20,32 \text { ans (1988) } \\
\mathrm{S}=\text { Échantillon d'étudiants de premier cycle } \\
\text { dans un cours d'introduction à la psychologie } \\
\mathrm{D}=\text { Transversales } \\
\mathrm{AD}=\text { Analyses de la variance, du test du chi } \\
\text { carré et log-linéaire multivariée } \\
\mathrm{T}=\text { Analytique }\end{array}$ & $\begin{array}{l}\text { Anglo-Celtiques [165]; } \\
\text { Nord-Européens [22]; } \\
\text { Sud-Européens [79]; } \\
\text { Européens de I'Est [36]; } \\
\text { Asiatiques de l'Est [25]; } \\
\text { Sud-Asiatiques [25] }\end{array}$ & $\begin{array}{l}\text { VI : Sexe et ethnicité } \\
\text { VD : Dépression (scores } \\
\text { totaux et par item pour le } \\
\text { BDI) }\end{array}$ & $\begin{array}{l}\text { 1. L'origine ethnique est établie d'après le nom } \\
\text { de famille à l'aide de plusieurs dictionnaires } \\
\text { de noms de famille. } \\
\text { 2. Cette façon de faire a limité la capacité } \\
\text { d'identifier les Noirs de milieux } \\
\text { ethnoculturels antillais et les Sud-Asiatiques } \\
\text { de milieux chrétiens. }\end{array}$ \\
\hline $\begin{array}{l}\text { 32. Bagley, } 1993 \\
\text { (Calgary, Alberta, } \\
\text { Canada et Kowloon, } \\
\text { Hong Kong, Chine) }^{58}\end{array}$ & $\begin{array}{l}\mathrm{N}=300 \\
\mathrm{~A}=60 \text { à } 74 \text { ans } \\
\mathrm{S}=\text { L'échantillon composé de } 100 \text { Chinois âgés } \\
\text { parlant le cantonnais et vivant au Canada a été } \\
\text { recruté au moyen d'une sélection aléatoire des } \\
\text { noms de familles chinois dans l'annuaire } \\
\text { téléphonique. Un premier appel a été effectué } \\
\text { par une personne parlant le cantonnais, qui a } \\
\text { constaté la présence d'une personne âgée (de } \\
60 \text { à } 74 \text { ans) et qui a ensuite demandé et établi } \\
\text { l'origine ethnique de cette personne âgée. Un } \\
\text { échantillon stratifié composé de personnes } \\
\text { jeunes à âgées habitant Kowloon, à Hong } \\
\text { Kong, correspondant au profil d'âge et de sexe } \\
\text { du groupe de chinois âgés parlant le } \\
\text { cantonnais et habitant au Canada a été utilisé. } \\
\mathrm{D}=\text { Transversales } \\
\mathrm{AD}=\text { Comparaison des scores normalisés } \\
\mathrm{T}=\text { Descriptive }\end{array}$ & $\begin{array}{l}\text { Personnes d'origine } \\
\text { européenne nées au Canada } \\
\text { [100]; Immigrants chinois } \\
\text { établis depuis longtemps au } \\
\text { Canada [50]; Immigrants } \\
\text { chinois nouvellement arrivés } \\
\text { au Canada [50]; Chinois de } \\
\text { Hong Kong [100] }\end{array}$ & $\begin{array}{l}\text { VD : Santé physique et } \\
\text { mentale telle que mesurée } \\
\text { par le questionnaire sur l'état } \\
\text { de santé général; solitude } \\
\text { selon l'échelle de mesure de } \\
\text { la solitude de l'UCLA; qualité } \\
\text { de vie et acculturation et } \\
\text { satisfaction globale de vivre }\end{array}$ & $\begin{array}{l}\text { 1. Une définition explicite de l'ethnicité est } \\
\text { fournie. } \\
\text { 2. Différentes techniques semblent avoir été } \\
\text { utilisées d'un groupe à l'autre pour établir } \\
\text { l'ethnicité : le nom de famille, la langue et } \\
\text { une question inconnue pour les Chinois } \\
\text { habitant au Canada; le pays de naissance } \\
\text { pour les Chinois de Hong Kong et une } \\
\text { méthode inconnue pour les } \\
\text { Euro-Canadiens. }\end{array}$ \\
\hline
\end{tabular}




\begin{tabular}{|c|c|c|c|c|}
\hline $\begin{array}{l}\text { Auteur(s), année de } \\
\text { publication et région } \\
\text { à l'étude }\end{array}$ & $\begin{array}{l}\text { Échantillon de l'étude (taille de } \\
\text { l'échantillon, source des données, } \\
\text { groupe d'âge inclus et méthodologie) }\end{array}$ & Groupes ethniques inclus & Phénomène étudié & Commentaires \\
\hline $\begin{array}{l}\text { 33. Dion, } 1996 \text { (Toronto, } \\
\text { Canada) }{ }^{59} \mathrm{~N}=950\end{array}$ & $\begin{array}{l}A=18 \text { à } 21 \text { ans (?) : étudiants de première } \\
\text { année d'université } \\
S=\text { Échantillon de commodité } \\
D=\text { Transversales } \\
A D=\text { Analyse de la variance } \\
T=\text { Exploratoire }\end{array}$ & $\begin{array}{l}\text { Langue comme indicateur de } \\
\text { l'origine ethnique } \\
\text { Anglais; Chinois; Européens }\end{array}$ & $\begin{array}{l}\text { VD : Alexithymie (TAS-20) et } \\
\text { trois facteurs sous-jacents } \\
\text { (DIF= difficulté à reconnaître } \\
\text { les sentiments, DDF= difficulté } \\
\text { à décrire les sentiments, EOT= } \\
\text { pensée orientée vers } \\
\text { l'extérieur) }\end{array}$ & $\begin{array}{l}\text { 1. Dion indique la langue comme variable de } \\
\text { substitution possible pour l'ethnicité (c.-à-d. } \\
\text { l'origine ethnique). } \\
\text { 2. Aucune définition de l'ethnicité n'est } \\
\text { fournie. }\end{array}$ \\
\hline $\begin{array}{l}\text { 34. Heine et Lehman, } \\
1999 \text { (Canada et }^{60} \\
\text { Japon) }\end{array}$ & $\begin{array}{l}N=402 \\
A=18 \text { à } 25 \text { ans? } \\
S=\text { Enquête auprès des étudiants universitaires } \\
D=\text { Transversales } \\
A D=\text { Analyse de la variance avec tests } \\
\text { post-hoc (test HSD de Tukey) } \\
T=\text { Analytique }\end{array}$ & $\begin{array}{l}\text { Japonais [161]; Canadiens } \\
\text { d'origine asiatique [151]; } \\
\text { Euro-Canadiens [90] }\end{array}$ & $\begin{array}{l}\text { VD : Traits de personnalité : } \\
20 \text { items permettant de cerner } \\
\text { la façon dont les personnes } \\
\text { évaluent leur soi actuel et leur } \\
\text { soi idéal et ce qui décrit, selon } \\
\text { elles, les traits de l'étudiant } \\
\text { moyen; la différence entre le } \\
\text { soi actuel et le soi idéal de ces } \\
\text { personnes, ainsi que } \\
\text { l'importance des traits en ce } \\
\text { qui concerne la réussite dans } \\
\text { leur pays ont aussi été } \\
\text { évalués; dépression (ZSDS) }\end{array}$ & $\begin{array}{l}\text { 1. Aucune définition explicite de l'ethnicité } \\
\text { n'est fournie; elle semble être établie } \\
\text { d'après la culture. } \\
\text { 2. La méthode employée pour choisir } \\
\text { l'échantillon n'est pas claire. } \\
\text { 3. Les hypothèses relatives au statut } \\
\text { socio-économique et au SS n'ont pas été } \\
\text { testées. }\end{array}$ \\
\hline $\begin{array}{l}\text { 35. Rousseau et coll., } \\
2001 \text { (Montréal, } \\
\text { Canada) }^{61}\end{array}$ & $\begin{array}{l}\mathrm{N}=113 \\
\mathrm{~A}=20 \text { à } 65 \text { ans } \\
\mathrm{S}=\text { Échantillon de réfugiés qui sont allés } \\
\text { chercher de l'aide auprès d'organismes } \\
\text { communautaires qui offrent des services aux } \\
\text { réfugiés ou aux immigrants dans la région de } \\
\text { Montréal } \\
\mathrm{D}=\text { Combinaison de données qualitatives et } \\
\text { d'un modèle transversal quantitatif } \\
\mathrm{AD}=\text { Analyses descriptives et de corrélation } \\
\text { des rangs de Spearman et tests t pour des } \\
\text { analyses comparatives } \\
\mathrm{T}=\text { Descriptive }\end{array}$ & $\begin{array}{l}\text { Latino-Américains [60]; } \\
\text { Afro-Américains [53] }\end{array}$ & $\begin{array}{l}\text { VD : Profil émotionnel des } \\
\text { sujets établi d'après le } \\
\text { SCL-90R; trouble de stress } \\
\text { post-traumatique selon le } \\
\text { DSM-IV }\end{array}$ & $\begin{array}{l}\text { 1. L'origine ethnique est établie en fonction de } \\
\text { la région géoculturelle de naissance. } \\
\text { 2. Aucune définition explicite de l'ethnicité } \\
\text { n'est fournie. }\end{array}$ \\
\hline $\begin{array}{l}\text { 36. Howard et coll., } \\
2003 \text { (Toronto, Ontario, } \\
\text { Canada; É.-U.; Taipei, } \\
\text { Taiwan) }^{62}\end{array}$ & $\begin{array}{l}\mathrm{N}=3030 \\
\mathrm{~A}=\text { Non précisé } \\
\mathrm{S}=\text { Tous les participants du Canada sont des } \\
\text { volontaires recrutés à Toronto grâce à une } \\
\text { annonce dans le journal ou à un dépliant; les } \\
\text { Taïwanais ont été recrutés au Taipei City } \\
\text { Psychiatric Center; les fumeurs Afro-Américains } \\
\text { ont été recrutés au University of Kansas } \\
\text { Medical Center (établissements cliniques et } \\
\text { communautaires) } \\
\mathrm{D}=\text { Analyse par association (transversales) } \\
\mathrm{AD}=\text { Analyse de la variance; analyses du test } \mathrm{t} \text {, } \\
\text { du test } \mathrm{U} \text { de Mann-Whitney et du test du chi } \\
\text { carré } \\
\mathrm{T}=\text { Analytique }\end{array}$ & $\begin{array}{l}\text { Au Canada : } \\
\text { Indo-Asiatiques [48]; Chinois } \\
\text { [210]; Japonais [128]; } \\
\text { Canadiens d'origine africaine } \\
\text { [58]; Autochtones [228]; } \\
\text { Caucasiens [1 734] } \\
\text { Ailleurs : } \\
\text { Taïwanais [420]; } \\
\text { Afro-Américains [204] }\end{array}$ & $\begin{array}{l}\text { VD : Allèle CYP2E1*1D; } \\
\text { inactivation de I'alcool }\end{array}$ & $\begin{array}{l}\text { 1. L'ethnicité n'est pas définie de façon } \\
\text { explicite. } \\
\text { 2. Le milieu ethnique est établi en fonction } \\
\text { des grands-parents de la personne; il s'agit } \\
\text { donc d'une conceptualisation en fonction } \\
\text { de l'origine ethnique. }\end{array}$ \\
\hline $\begin{array}{l}\text { 37. Tweed et coll., } 2004 \\
\text { (Colombie-Britannique, } \\
\text { Canada et Japon) }^{63}\end{array}$ & $\begin{array}{l}\mathrm{N}=123 \text { (Étude } 1 \text { ) } \\
\quad 415 \text { (Étude 2) } \\
\mathrm{A}=18 \text { à } 47 \text { ans } \\
\mathrm{S}=\text { Étudiants de premier cycle de l'Université } \\
\text { de la Colombie-Britannique, Canada, et de } \\
\text { I'Université Ritsumeikan (Étude } 1 \text { ) ou de } \\
\text { I'Université Kurume (Étude 2), Japon } \\
\mathrm{D}=\text { Transversales } \\
\mathrm{AD}=\text { Analyse de la covariance multivariée } \\
\mathrm{T}=\text { Analytique }\end{array}$ & $\begin{array}{l}\text { Étude } 1 \text { : } \\
\text { Canadiens originaires de } \\
\text { I'Europe de l'Ouest [22]; } \\
\text { Canadiens originaires de } \\
\text { I'Asie de l'Est [57]; Canadiens } \\
\text { d'origine sud-asiatique ou } \\
\text { d'origine mixte [18]; Japonais } \\
\text { [26] } \\
\text { Étude 2: } \\
\text { Euro-Canadiens [68]; } \\
\text { Canadiens originaires de } \\
\text { l'Asie de l'Est [106]; Japonais } \\
\text { [241] }\end{array}$ & $\begin{array}{l}\text { VD : Événements stressants et } \\
\text { négatifs de la vie; habiletés à } \\
\text { faire face au stress selon la } \\
\text { WCCL et les éléments de } \\
\text { réaction au stress des } \\
\text { Japonais }\end{array}$ & $\begin{array}{l}\text { 1. L'ethnicité n'est pas définie de façon } \\
\text { explicite, mais elle est établie en fonction } \\
\text { de la culture. } \\
\text { 2. L'ethnicité est conceptualisée selon l'origine, } \\
\text { donc selon l'origine ethnique. }\end{array}$ \\
\hline
\end{tabular}




\begin{tabular}{|c|c|c|c|c|}
\hline $\begin{array}{l}\text { Auteur(s), année de } \\
\text { publication et région } \\
\text { à l'étude }\end{array}$ & $\begin{array}{l}\text { Échantillon de l'étude (taille de } \\
\text { l'échantillon, source des données, } \\
\text { groupe d'âge inclus et méthodologie) }\end{array}$ & Groupes ethniques inclus & Phénomène étudié & Commentaires \\
\hline $\begin{array}{l}\text { 38. Khanlou, } 2004 \\
\text { (Hamilton, Ontario, } \\
\text { Canada) }^{64}\end{array}$ & $\begin{array}{l}\mathrm{N}=550 \\
\mathrm{~A}=\text { de la } 9^{\mathrm{e}} \text { à la } 13^{e} \text { année } \\
\mathrm{S}=\text { Enquête sur des élèves de } 4 \text { écoles } \\
\text { secondaires de la région de Hamilton } \\
\text { Wentworth, menée en } 1998 \\
\mathrm{D}=\text { Transversales } \\
\mathrm{AD}=\text { Analyse du test du chi carré et analyse de } \\
\text { la variance } \\
\mathrm{T}=\text { Descriptive }\end{array}$ & $\begin{array}{l}\text { Information donnée au sujet } \\
\text { des trois milieux ethniques ou } \\
\text { culturels auxquels } \\
\text { appartiennent le plus } \\
\text { fréquemment la mère et le } \\
\text { père des élèves. } \\
\text { Selon la mère : } \\
\text { Italiens [48]; } \\
\text { Portugais [43]; } \\
\text { Irlandais [35]; } \\
\text { Anglais [35] } \\
\text { Selon le père : } \\
\text { Italiens [69]; } \\
\text { Portugais [42]; } \\
\text { Canadiens [38] }\end{array}$ & $\begin{array}{l}\text { VD : Estime de soi selon les } \\
\text { échelles RSE et CSE }\end{array}$ & $\begin{array}{l}\text { 2. L'ethnicité est établie selon le " milieu } \\
\text { ethnique ou culturel d'origine des parents * } \\
\text { [Traduction], indiquant une } \\
\text { conceptualisation en fonction de l'origine } \\
\text { ethnique. } \\
\text { 3. La division en groupes ethniques } \\
\text { spécifiques a été utilisée, et la taille de } \\
\text { l'échantillon de certains de ces groupes } \\
\text { était très petite. Par conséquent, seules les } \\
\text { trois catégories ethniques les plus } \\
\text { importantes ont été comparées. }\end{array}$ \\
\hline $\begin{array}{l}\text { 39. Aubert et coll., } 2004 \\
\text { (Canada) }^{65}\end{array}$ & $\begin{array}{l}\mathrm{N}=170 \\
\mathrm{~A}=18 \text { ans et plus } \\
\mathrm{S}=\text { Échantillon de commodité composé } \\
\text { d'étudiants } \\
\mathrm{D}=\text { Transversales } \\
\mathrm{AD}=\text { Analyse de la variance } \\
\mathrm{T}=\text { Analytique }\end{array}$ & $\begin{array}{l}\text { Autres Canadiens [81]; } \\
\text { Canadiens d'origine chinoise } \\
\text { [89] }\end{array}$ & $\begin{array}{l}\text { VD : Hostilité (HDHQ); } \\
\text { probabilité que la personne se } \\
\text { suicide (SPS); comportement } \\
\text { agressif permanent et } \\
\text { tentative de suicide; pensées } \\
\text { suicidaires et automutilation } \\
\text { délibérée }\end{array}$ & $\begin{array}{l}\text { 1. L'ethnicité a été examinée en tant que } \\
\text { covariable. } \\
\text { 2. On ne sait pas exactement si le groupe des } \\
\text { " autres Canadiens " comprend des ethnies } \\
\text { autres que les Canadiens anglais. } \\
\text { 3. L'origine ethnique est établie en fonction du } \\
\text { pays de naissance. }\end{array}$ \\
\hline \multicolumn{5}{|c|}{ Études de moindre envergure impliquant une collecte de données brutes - Échantillon clinique ou spécialisé (n=10) } \\
\hline $\begin{array}{l}\text { 40. Bland et Orn, } 1981 \\
\text { (Alberta, Canada) }^{66}\end{array}$ & $\begin{array}{l}\mathrm{N}=43 \text { ( } 33 \text { non-immigrants et } 10 \text { immigrants) } \\
\mathrm{A}=15 \text { à } 49 \text { ans } \\
\mathrm{S}=\text { Données sur l'hospitalisation pour une } \\
\text { première crise en } 1963 \\
\mathrm{D}=\text { Suivi rétrospectif d'une cohorte composée } \\
\text { de personnes atteintes de schizophrénie } \\
\text { comparée à la population générale } \\
\mathrm{AD}=\text { Analyses descriptive et du test du chi } \\
\text { carré } \\
\mathrm{T}=\text { Descriptive }\end{array}$ & $\begin{array}{l}10 \text { immigrants classifiés en } \\
\text { tant que " autres Européens " } \\
\text { [6] et Européens de l'Est [4] }\end{array}$ & VD : Schizophrénie & $\begin{array}{l}\text { 1. L'ethnicité a été examinée en tant que } \\
\text { covariable. } \\
\text { 2. Le terme " groupe ethnique " a été employé, } \\
\text { mais aucune définition de ce qu'il signifie } \\
\text { n'est fournie. } \\
\text { 3. L'origine ethnique est établie selon le lieu de } \\
\text { naissance. }\end{array}$ \\
\hline $\begin{array}{l}\text { 41. Seltzer et Langford, } \\
1984 \text { (Territoires du } \\
\text { Nord-Ouest, Canada) }^{67}\end{array}$ & $\begin{array}{l}\mathrm{N}=85 \\
\mathrm{~A}=15 \text { à } 25 \text { ans } \\
\mathrm{S}=\text { Échantillon de commodité (c.-à-d. toutes les } \\
\text { personnes dirigées par un tribunal ou un } \\
\text { conseiller juridique au Département de } \\
\text { psychiatrie de l'hôpital régional au cours de } \\
\text { l'année civile 1981) } \\
\mathrm{D}=\text { Transversales } \\
\mathrm{AD}=\text { Descriptive } \\
\mathrm{T}=\text { Descriptive }\end{array}$ & $\begin{array}{l}\text { Inuits [41]; Métis/Dénés [27]; } \\
\text { Caucasiens [17] }\end{array}$ & $\begin{array}{l}\text { VD : Diagnostic psychiatrique } \\
\text { issu du DSM-III et type } \\
\text { d'infraction criminelle } \\
\text { commise }\end{array}$ & $\begin{array}{l}\text { 1. Aucune définition explicite de l'ethnicité } \\
\text { n'est fournie. } \\
\text { 2. Le groupe des Autochtones a été divisé en } \\
\text { deux (Inuits et Métis), ce qui indique des } \\
\text { particularités culturelles, mais le groupe des } \\
\text { Caucasiens n'a pas été désagrégé. }\end{array}$ \\
\hline $\begin{array}{l}\text { 42. Borzecki et coll., } \\
1988 \text { (Saskatoon, } \\
\text { Saskatchewan, } \\
\text { Canada) }^{68}\end{array}$ & $\begin{array}{l}\mathrm{N}=275 \text { (tous de sexe masculin) } \\
\mathrm{A}=28,41 \text { = âge moyen à l'admission } \\
\mathrm{S}=1^{\text {re }} \text { admission successive à l'installation } \\
\text { entre janvier } 1978 \text { et septembre } 1982 \\
\mathrm{D}=\text { Transversales } \\
\mathrm{AD}=\text { Analyse de la variance, analyse de la } \\
\text { covariance et coefficient de corrélation de } \\
\text { Pearson } \\
T=\text { Analytique }\end{array}$ & $\begin{array}{l}\text { Autochtones (c.-à-d. Inuits, } \\
\text { Métis et Amérindiens) [57]; } \\
\text { Non-Autochtones [218] }\end{array}$ & $\begin{array}{l}\text { VD : Profil psychologique } \\
\text { établi selon le MMPI }\end{array}$ & $\begin{array}{l}\text { 1. Aucune définition explicite de l'ethnicité } \\
\text { n'est fournie. } \\
\text { 2. L'ethnicité est établie selon les origines } \\
\text { ancestrales (origine ethnique), du moins pour } \\
\text { l'échantillon des Autochtones, alors que } \\
\text { tous les non-Autochtones sont groupés } \\
\text { ensemble, et ce, malgré l'hétérogénéité de } \\
\text { leurs origines ancestrales. }\end{array}$ \\
\hline $\begin{array}{l}\text { 43. Chandrasena et } \\
\text { coll., } 1991 \text { (Ontario, } \text { Canada) }^{69}\end{array}$ & $\begin{array}{l}\mathrm{N}=117 \\
\mathrm{~A}=\text { Groupe d'âge non précisé } \\
\mathrm{S}=\text { L'ensemble des suicides s'étant produits } \\
\text { dans trois établissements psychiatriques de } \\
\text { I'Ontario entre } 1967 \text { et } 1990 \\
\mathrm{D}=\text { Transversales } \\
\mathrm{AD}=\text { Analyses descriptives } \\
\mathrm{T}=\text { Descriptive }\end{array}$ & $\begin{array}{l}\text { Personnes nées au Canada } \\
\text { [94]; Personnes nées à } \\
\text { l'étranger [23] }\end{array}$ & VD : Suicide & $\begin{array}{l}\text { 1. L'ethnicité est mentionnée, mais elle n'est } \\
\text { pas définie. } \\
\text { 2. Le fait d'étudier les personnes nées au } \\
\text { Canada par rapport aux personnes nées à } \\
\text { l'étranger indique une conceptualisation en } \\
\text { fonction du pays de naissance, quoique ce } \\
\text { ne soit pas mentionné explicitement. }\end{array}$ \\
\hline $\begin{array}{l}\text { 44. Norton et coll., } 1995 \\
\text { (Manitoba, Canada) }^{70}\end{array}$ & $\begin{array}{l}\mathrm{N}=80 \\
\mathrm{~A}=\text { ? } \\
\mathrm{S}=\text { Participants à la Fondation manitobaine de } \\
\text { lutte contre les dépendances } \\
\mathrm{D}=\text { Transversales } \\
\mathrm{AD}=\text { Test du chi carré } \\
\mathrm{T}=\text { Descriptive }\end{array}$ & $\begin{array}{l}\text { Autochtones du Canada [37]; } \\
\text { Anglo-Canadiens [43] }\end{array}$ & $\begin{array}{l}\text { VD : Idées suicidaires (selon } \\
\text { les enquêtes } \\
\text { épidémiologiques des zones } \\
\text { de recrutement du NIMH), } \\
\text { panique (PAQ), abus de } \\
\text { drogues chimiques (bMAST et } \\
\text { TDAD) et dépression (BDI) }\end{array}$ & $\begin{array}{l}\text { 1. Aucune définition explicite de l'ethnicité } \\
\text { n'est fournie. } \\
\text { 2. On ne sait pas exactement comment } \\
\text { l'ethnicité a été déterminée. }\end{array}$ \\
\hline
\end{tabular}




\begin{tabular}{|c|c|c|c|c|}
\hline $\begin{array}{l}\text { Auteur(s), année de } \\
\text { publication et région } \\
\text { à l'étude }\end{array}$ & $\begin{array}{l}\text { Échantillon de l'étude (taille de } \\
\text { l'échantillon, source des données, } \\
\text { groupe d'âge inclus et méthodologie) }\end{array}$ & Groupes ethniques inclus & Phénomène étudié & Commentaires \\
\hline $\begin{array}{l}\text { 45. Weekes et coll., } \\
1995 \text { (Ontario, }^{\text {Canada) }}{ }^{71}\end{array}$ & $\begin{array}{l}\mathrm{N}=301 \\
\mathrm{~A}=18 \text { à } 59 \text { ans } \\
\mathrm{S}=\text { Échantillon de commodité }: \text { Hommes } \\
\text { adultes incarcérés dans une prison fédérale à } \\
\text { sécurité moyenne } \\
\mathrm{D}=\text { Transversales } \\
\mathrm{AD}=\text { Analyse de la variance multivariée, du } \\
\text { test } \mathrm{t} \text {, de corrélation et des composantes } \\
\text { principales } \\
\mathrm{T}=\text { Analytique }\end{array}$ & $\begin{array}{l}\text { Caucasiens [203]; } \\
\text { Autochtones [59]; } \\
\text { Métis [39] }\end{array}$ & $\begin{array}{l}\text { VD : Psychopathologie selon } \\
\text { le MCMI }\end{array}$ & $\begin{array}{l}\text { 1. Aucune définition explicite de l'ethnicité. } \\
\text { 2. Les termes " ethnicité ", " groupe culturel " } \\
\text { et " identification raciale " ont été employés } \\
\text { dans cet article, mais la catégorisation } \\
\text { repose sur "l'identification raciale " } \\
\text { déclarée par les personnes. }\end{array}$ \\
\hline $\begin{array}{l}\text { 46. Pawliuk et coll., } \\
1996 \text { (Montréal, } \\
\text { Canada) }^{72}\end{array}$ & $\begin{array}{l}\mathrm{N}=34 \text { familles multiethniques } \\
\text { (monoparentales incluses) avec un total de } \\
48 \text { enfants (dont } 8 \text { sont nés en Asie et } 3 \text { en } \\
\text { Inde. Il n'y a aucune autre mention de la } \\
\text { division ethnique des enfants.) } \\
\mathrm{A}=\text { La groupe d'âge des parents n'est pas } \\
\text { précisé, mais les enfants sont âgés de } 6,5 \text { à } \\
17 \text { ans. } \\
\mathrm{S}=\text { Échantillon de commodité composé de } \\
\text { parents et de leurs enfants vus dans une } \\
\text { clinique pédiatrique de Montréal, Canada } \\
\mathrm{D}=\text { Transversales } \\
\mathrm{AD}=\text { Analyse de la variance et analyse de la } \\
\text { variance multivariée } \\
\mathrm{T}=\text { Analytique }\end{array}$ & $\begin{array}{l}\text { La division ethnique des } \\
\text { parents est fournie, mais pas } \\
\text { celle des enfants. } \\
\text { Asiatiques [26]; } \\
\text { Européens [5]; } \\
\text { Indiens/Sud-Américains/ } \\
\text { Moyen-Orientaux [3] }\end{array}$ & $\begin{array}{l}\text { VD : le CBCL révisé; l'échelle } \\
\text { d'auto-évaluation de la } \\
\text { dépression; la What I Think } \\
\text { and Feel Scale; la Children's } \\
\text { Psychosomatic Symptom } \\
\text { Checklist et la Hare } \\
\text { Self-Esteem Scale. }\end{array}$ & $\begin{array}{l}\text { 1. Aucune définition explicite de l'ethnicité } \\
\text { n'est fournie } \\
\text { 2. Il y a } 34 \text { parents et } 48 \text { enfants; on ne sait } \\
\text { pas exactement quelle est la division de } \\
\text { l'ethnicité chez les enfants. } \\
\text { 3. Étant donné que l'ethnicité est fournie pour } \\
\text { les parents, mais non pour les enfants, il } \\
\text { semble que l'ethnicité soit fondée sur la } \\
\text { région ou le lieu de naissance des parents. }\end{array}$ \\
\hline $\begin{array}{l}\text { 47. Zapf et coll., } 1996 \\
\text { (Vancouver, } \\
\text { Colombie-Britannique, } \\
\text { Canada) }^{73}\end{array}$ & $\begin{array}{l}N=790 \\
A=\text { Non précisé } \\
S=\text { Hommes adultes choisis de façon aléatoire } \\
\text { au Pretrial Service Centre de Vancouver entre le } \\
1^{\text {er }} \text { août } 1989 \text { et le } 31 \text { juillet } 1990 \\
D=\text { Transversales } \\
A D=\text { Analyses du test du chi carré et du } \\
\text { coefficient de corrélation de Pearson } \\
T=\text { Descriptive }\end{array}$ & $\begin{array}{l}\text { Bien que le terme " ethnicité " } \\
\text { ait été employé, il n'y a } \\
\text { aucune division des } \\
\text { participants en fonction de } \\
\text { leur groupe ethnique. }\end{array}$ & $\begin{array}{l}\text { VD : Trouble mental selon la } \\
\text { BPRS et le profil diagnostique }\end{array}$ & $\begin{array}{l}\text { 1. L'ethnicité a été mentionnée en tant que } \\
\text { covariable. } \\
\text { 2. Aucune définition de l'ethnicité n'est } \\
\text { fournie. } \\
\text { 3. Il n'y a aucune indication de la façon dont } \\
\text { l'ethnicité est conceptualisée et } \\
\text { opérationnalisée. }\end{array}$ \\
\hline $\begin{array}{l}\text { 48. Devins et coll., } 2000 \\
\text { (Canada et É.-U.) }^{74}\end{array}$ & $\begin{array}{l}\mathrm{N}=405 \\
\mathrm{~A}=\text { Non précisé } \\
\mathrm{S}=\text { L'Arthritis, Rheumatism and Aging Medical } \\
\text { Information System Lupus Project } \\
\mathrm{D}=\text { Longitudinales } \\
\mathrm{AD}=\text { Analyse des composantes principales et } \\
\text { des pistes causales } \\
\mathrm{T}=\text { Analytique }\end{array}$ & $\begin{array}{l}\text { Blanches [335]; } \\
\text { Noires [40]; } \\
\text { Asiatiques [30] (toutes de } \\
\text { sexe féminin) }\end{array}$ & $\begin{array}{l}\text { VD : Bien-être psychologique } \\
\text { (ABS); incapacité apprise (RAI); } \\
\text { trouble émotionnel (CES-D); } \\
\text { douleur musculo-squelettique } \\
\text { (questionnaire d'évaluation de } \\
\text { l'état de santé); bien-être } \\
\text { psychosocial global }\end{array}$ & $\begin{array}{l}\text { 1. Les groupes des Noires et des Asiatiques } \\
\text { sont principalement composés (c.-à-d. à } \\
97 \text { et à } 83 \% \text { d'Américaines, alors que le } \\
\text { groupe des Blanches est composé à parts } \\
\text { presque égales d'Américaines et de } \\
\text { Canadiennes. } \\
\text { 2. Il est question de classification raciale plutôt } \\
\text { que d'ethnicité. }\end{array}$ \\
\hline $\begin{array}{l}\text { 49. Hodelet, } 2001 \\
\text { (Colombie-Britannique, } \\
\text { Canada) }^{75}\end{array}$ & $\begin{array}{l}\mathrm{N}=175 \\
\mathrm{~A}=19 \text { à } 75 \text { ans } \\
\mathrm{S}=\text { Hôpital de psychiatrie légale sécurisé, tous } \\
\text { les dossiers de cas de patients hospitalisés } \\
\text { entre le } 1^{\text {er }} \text { décembre } 1998 \text { et le } \\
28 \text { février } 1999 \\
D=\text { Transversales } \\
A D=\text { Analyse du test du chi carré et analyse de } \\
\text { la variance } \\
T=\text { Descriptive }\end{array}$ & $\begin{array}{l}\text { Blancs [153]; } \\
\text { Amérindiens [26]; } \\
\text { Orientaux/Asiatiques } \\
\text { de l'Est [11]; } \\
\text { Sud-Asiatiques [5]; } \\
\text { Noirs [1] }\end{array}$ & $\begin{array}{l}\text { VD : Type d'infraction; type de } \\
\text { diagnostic psychiatrique; } \\
\text { psychose; tendance } \\
\text { psychotique }\end{array}$ & $\begin{array}{l}\text { 1. Une définition explicite de l'ethnicité n'est } \\
\text { pas fournie. } \\
\text { 2. L' « origine ethnique * est indiquée, mais on } \\
\text { ne sait pas exactement comment elle a été } \\
\text { déterminée dans les dossiers médicaux des } \\
\text { participants. }\end{array}$ \\
\hline
\end{tabular}

